\title{
On the interval of fluctuation of the singular values of random matrices
}

\author{
Olivier Guédon ${ }^{1} \quad$ Alexander E. Litvak ${ }^{2} \quad$ Alain Pajor \\ Nicole Tomczak-Jaegermann ${ }^{3}$
}

\begin{abstract}
Let $A$ be a matrix whose columns $X_{1}, \ldots, X_{N}$ are independent random vectors in $\mathbb{R}^{n}$. Assume that the tails of the 1-dimensional marginals decay as $\mathbb{P}\left(\left|\left\langle X_{i}, a\right\rangle\right| \geq t\right) \leq t^{-p}$ uniformly in $a \in S^{n-1}$ and $i \leq N$. Then for $p>4$ we prove that with high probability $A / \sqrt{n}$ has the Restricted Isometry Property (RIP) provided that Euclidean norms $\left|X_{i}\right|$ are concentrated around $\sqrt{n}$. We also show that the covariance matrix is well approximated by the empirical covariance matrix and establish corresponding quantitative estimates on the rate of convergence in terms of the ratio $n / N$. Moreover, we obtain sharp bounds for both problems when the decay is of the type $\exp \left(-t^{\alpha}\right)$ with $\alpha \in(0,2]$, extending the known case $\alpha \in[1,2]$.
\end{abstract}

AMS 2010 Classification: primary 60B20, 46B06, 15B52; secondary 46B09, $60 \mathrm{D} 05$

Keywords: random matrices, norm of random matrices, approximation of covariance matrices, compressed sensing, restricted isometry property, log-concave random vectors, concentration inequalities, deviation inequalities, heavy tails, spectrum, singular values, order statistics.

\footnotetext{
${ }^{1}$ The research is partially supported by the ANR GeMeCoD, ANR 2011 BS01 00701

${ }^{2}$ Research partially supported by the E.W.R. Steacie Memorial Fellowship.

${ }^{3}$ This author holds the Canada Research Chair in Geometric Analysis.
} 


\section{Introduction and main results}

Fix positive integers $n, N$ and let $A$ be an $n \times N$ random matrix whose columns $X_{1}, \ldots, X_{N}$ are independent random vectors in $\mathbb{R}^{n}$. For a subset $I \subset\{1, \ldots, N\}$ of cardinality $m$, denote by $A^{I}$ the $n \times m$ matrix whose columns are $X_{i}, i \in I$. We are interested in estimating the interval of fluctuation of the spectrum of some matrices related to $A$ when the random vectors $X_{i}, i \leq N$ have heavy tails; firstly, uniform estimates of the spectrum of $\left(A^{I}\right)^{\top} A^{I}$ which is the set of squares of the singular values of $A^{I}$, where $I$ runs over all subsets of cardinality $m$ for some fixed parameter $m$ and secondly estimates for the spectrum of $A A^{\top}$. The first problem is related to the notion of Restricted Isometry Property (RIP) with $m$ a parameter of sparsity whereas the second is about approximation of a covariance matrix by empirical covariance matrices.

These questions have been substantially developed over recent years and many papers devoted to these notions were written. In this work, we say that a random vector $X$ in $\mathbb{R}^{n}$ satisfies the tail behavior $\mathbf{H}(\phi)$ with parameter $\tau \geq 1$, if

$$
\mathbf{H}(\phi): \quad \forall a \in S^{n-1} \forall t>0 \quad \mathbb{P}(|\langle X, a\rangle| \geq t) \leq \tau / \phi(t)
$$

for a certain function $\phi$ and we assume that $X_{i}$ satisfies $\mathbf{H}(\phi)$ for all $i \leq N$. We will focus on two choices of the function $\phi$, namely $\phi(t)=t^{p}$, with $p>4$, which means heavy tail behavior for marginals, and $\phi(t)=(1 / 2) \exp \left(t^{\alpha}\right)$, with $\alpha \in(0,2]$, which corresponds to an exponential power type tail behavior and extends the known subexponential case $(\alpha=1$, see [2, 3]).

The concept of the Restricted Isometry Property was introduced in [10] in order to study an exact reconstruction problem by $\ell_{1}$ minimization algorithm, classical in compressed sensing. Although it provided only a sufficient condition for the reconstruction, it played a decisive role in the development of the theory, and it is still an important property. This is mostly due to the fact that a large number of important classes of random matrices have RIP. It is also noteworthy that the problem of reconstruction can be reformulated in terms of convex geometry, namely in terms of neighborliness of the symmetric convex hull of $X_{1}, \ldots, X_{N}$, as was shown in [12].

Let us recall the intuition of RIP (for the definition see (9) below). For an $n \times N$ matrix $T$ and $1 \leq m \leq N$, the isometry constant of order $m$ of $T$ is the parameter $0<\delta_{m}(T)<1$ such that the square of Euclidean norms $|T z|$ and $|z|$ are approximately equal, up to a factor $1+\delta_{m}(T)$, for all $m$-sparse 
vectors $z \in \mathbb{R}^{N}$ (that is, $|\operatorname{supp}(z)| \leq m$ ). Equivalently, this means that for every $I \subset\{1, \ldots, N\}$ with $|I| \leq m$, the spectrum of $\left(T^{I}\right)^{\top} T^{I}$ is contained in the interval $\left[1-\delta_{m}(T), 1+\delta_{m}(T)\right]$. In particular when $\delta_{m}(T)<\theta$ for small $\theta$, then the squares of singular values of the matrices $T^{I}$ belong to $[1-\theta, 1+\theta]$. Note that in compressed sensing for the reconstruction of vectors by $\ell_{1}$ minimization, one does not need RIP for all $\theta>0$ (see [12] and [11]). The RIP contains implicitly a normalization, in particular it implies that the Euclidean norms of the columns belong to an interval centered around one.

Let $A$ be an $n \times N$ random matrix whose columns are $X_{1}, \ldots, X_{N}$. In view of the example of matrices with i.i.d. entries, centered and with variance one, for which $\mathbb{E}\left|X_{i}\right|^{2}=n$, we normalized the matrix by considering $A / \sqrt{n}$ and we introduce the concentration function

$$
P(\theta):=\mathbb{P}\left(\max _{i \leq N}\left|\frac{\left|X_{i}\right|^{2}}{n}-1\right| \geq \theta\right) .
$$

Until now the only known cases of random matrices satisfying a RIP were the cases of subgaussian [9, 10, 12, 23] and subexponential [4] matrices. Our first main theorem says that matrices we consider have the RIP of order $m$, with "large" $m$ of the form $m=n \psi(n / N)$ with $\psi$ depending on $\phi$ and possibly on other parameters. In particular, when $N$ is proportional to $n$, then $m$ is proportional to $n$. We present a simplified version of our result, for the detailed version see Theorem 3.1 below.

Theorem 1.1 Let $0<\theta<1$. Let $A$ be a random $n \times N$ matrix whose columns $X_{1}, \ldots, X_{N}$ are independent random vectors satisfying hypothesis $\mathbf{H}(\phi)$ for some $\phi$. Assume that $n, N$ are large enough. Then there exists a function $\psi$ depending on $\phi$ and $\theta$ such that with high probability (depending on the concentration function $P(\theta)$ ) the matrix $A / \sqrt{n}$ has $R I P$ of order $m=$ $[n \psi(n / N)]$ with a parameter $\theta$ (that is, $\delta_{m}(A / \sqrt{n}) \leq \theta$ ).

The second problem we investigate goes back to a question of Kannan, Lovász and Simonovits (KLS). As before assume that $A$ is a random $n \times N$ matrix whose columns $X_{1}, \ldots, X_{N}$ are independent random vectors satisfying hypothesis $\mathbf{H}(\phi)$ for some $\phi$. Additionally assume that $X_{i}$ 's are identically distributed as a centered random vector $X$. KLS question asks how fast the empirical covariance matrix $U:=(1 / N) A A^{\top}$ converges to the covariance matrix $\Sigma:=(1 / N) \mathbb{E} A A^{\top}=\mathbb{E} U$. Of course this depends on assumptions on $X$. In particular, is it true that with high probability the operator norm 
$\|U-\Sigma\| \leq \varepsilon\|\Sigma\|$ for $N$ being proportional to $n$ ? Originally this was asked for log-concave random vectors but the general question of approximating the covariance matrix by sample covariance matrices is an important subject in Statistics as well as on its own right. The corresponding question in Random Matrix Theory is about the limit behavior of smallest and largest singular values. In the case of Wishart matrices, that is when the coordinates of $X$ are i.i.d. centered random variables of variance one, the Bai-Yin theorem [6] states that under assumption of boundedness of fourth moments the limits of minimal and maximal singular numbers of $U$ are $(1 \pm \sqrt{\beta})^{2}$ as $n, N \rightarrow \infty$ and $n / N \rightarrow \beta \in(0,1)$. Moreover, it is known [7, 30] that boundedness of fourth moment is necessary in order to have the convergence of the largest singular value. The asymptotic non-limit behavior (also called "non-asymptotic" in Statistics), i.e., sharp upper and lower bounds for singular values in terms of $n$ and $N$, when $n$ and $N$ are sufficiently large, was studied in several works. To keep the notation more compact and clear we put

$$
M:=\max _{i \leq N}\left|X_{i}\right|, \quad S:=\sup _{a \in S^{n-1}}\left|\frac{1}{N} \sum_{i=1}^{N}\left(\left\langle X_{i}, a\right\rangle^{2}-\mathbb{E}\left\langle X_{i}, a\right\rangle^{2}\right)\right| .
$$

Note that if $\mathbb{E}\langle X, a\rangle^{2}=1$ for every $a \in S^{n-1}$ (that is, $X$ is isotropic), then the bound $S \leq \varepsilon$ is equivalent to the fact that the singular values of $U$ belong to the interval $[1-\varepsilon, 1+\varepsilon]$. For Gaussian matrices it is known $([13,32])$ that with probability close to one

$$
S \leq C \sqrt{n / N}
$$

where $C$ is a positive absolute constant. In [2, 3] the same estimate was obtained for a large class of random matrices, which in particular did not require that entries of the columns are independent, or that $X_{i}$ 's are identically distributed. In particular this solved the original KLS problem. More precisely, (4) holds with high probability under the assumptions that the $X_{i}$ 's satisfy hypothesis $\mathbf{H}(\phi)$ with $\phi(t)=e^{t} / 2$ and that $M \leq C(N n)^{1 / 4}$ with high probability. Both conditions hold for log-concave random vectors.

Until recent time, quite strong conditions on the tail behavior of the one dimensional marginals of the $X_{i}$ were imposed, typically of subexponential type. Of course, in view of Bai-Yin theorem, it is a natural question whether one can replace the function $\phi(t)=e^{t} / 2$ by the function $\phi(t)=e^{t^{\alpha}} / 2$ with $\alpha \in(0,1)$ or $\phi(t)=t^{p}$, for $p \geq 4$. The first attempt in this direction was done 
in [34], where the bound $S \leq C(p, K)(n / N)^{1 / 2-2 / p}(\ln \ln n)^{2}$ was obtained for every $p>4$ provided that $M \leq K \sqrt{n}$. Clearly, $\ln \ln n$ is a "parasitic" term, which, in particular, does not allow to solve the KLS problem with $N$ proportional to $n$. This problem was solved in [24, 31] under strong assumptions and in particular when $M \leq K \sqrt{n}$ and $X$ has i.i.d. coordinates with bounded $p$-th moment with $p>4$. Very recently, in [25, the "right" upper bound $S \leq C(n / N)^{1 / 2}$ was proved for $p>8$ provided that $M \leq$ $C(N n)^{1 / 4}$. The methods used in 25] play an influential role in the present paper.

The problems of estimating the smallest and the largest singular values are quite different. One expects weaker assumption for estimating the smallest singular value. This already appeared in the work [31] and was pushed further in recent works [19, 33, 35] and in [14, 20, 26] which led to new bounds on the performance of $\ell_{1}$-minimization methods.

In this paper we solve the KLS problem for $4<p \leq 8$, in Theorem 1.2. Our argument works also in other cases and makes the bridge between the known cases $p>8$ and the exponential case.

Theorem 1.2 Let $X_{1}, \ldots, X_{N}$ be independent random vectors in $\mathbb{R}^{n}$ satisfying hypothesis $\mathbf{H}(\phi)$ with $\phi(t)=t^{p}$ for some $p \in(4,8]$. Let $\varepsilon \in(0,1)$ and $\gamma=p-4-2 \varepsilon>0$. Then

$$
S \leq C\left(\left(\frac{M^{2}}{n}\right)\left(\frac{n}{N}\right)+C(p, \varepsilon)\left(\frac{n}{N}\right)^{\gamma / p}\right)
$$

with probability larger than $1-8 e^{-n}-2 \varepsilon^{-p / 2} \max \left\{N^{-3 / 2}, n^{-(p / 4-1)}\right\}$.

In particular, if $N$ is proportional to $n$ and $M^{2} / n$ is bounded by a constant with high probability, which is the case for large classes of random vectors, then with high probability

$$
S \leq C(n / N)^{\gamma / p}
$$

Let $X$ have i.i.d. coordinates distributed as a centered random variable with finite $p$-th moment, $p>2$. Then by Rosenthal's inequality ([29], see also [17] and Lemma 6.3 below), $X$ satisfies hypothesis $\mathbf{H}(\phi)$ with $\phi(t)=t^{p}$. Let $X_{1}, \ldots, X_{N}$ be independent random vectors distributed as $X$. It is known (77], 30], see also [22] for a quantitative version) that when $N$ is proportional to $n$ and in the absence of fourth moment, $M^{2} / n \rightarrow \infty$ as $n \rightarrow \infty$. Hence, 
bounds for $S$ involving the term $M^{2} / n$ like the bound (5) are of interest only for $p \geq 4$. We don't know if it holds in the case $p=4$.

The main novelty of our proof is a delicate analysis of the behavior of norms of submatrices, namely quantities $A_{k}$ and $B_{k}, k \leq N$, defined in (6) below. This analysis is done in Theorem 2.1, which is in the heart of the technical part of the paper and it will be presented in the next section. The estimates for $B_{k}$ are responsible for RIP, Theorem 1.1, while the estimates for $A_{k}$ are responsible for KLS problem, Theorem 1.2 .

As usual in this paper $C, C_{0}, C_{1}, \ldots, c, c_{0}, c_{1}, \ldots$ always denote absolute positive constants whose values may vary from line to line.

The paper is organized as follows. In Section 2, we formulate the main technical result. For the reader convenience, we postpone its proof till Section 5. In Section 3, we discuss the results on RIP. The fully detailed formulation of the main result in this direction is Theorem 3.1, while Theorem 1.1 is its very simplified corollary. In Section 4 , we prove Theorem 1.2 as a consequence of Theorem 4.5. The case $p>8$ and the exponential cases are proved in Theorem 4.7 using the same argument. Symmetrization and formulas for sums of the $k$ smallest order statistics of independent non-negative random variables with heavy tails allow to reduce the problem on hand to estimates for $A_{k}$. In the last Section [6, we discuss optimality of the results.

An earlier version of the main results of this paper was announced in [15].

Acknowledgment. A part of this research was performed while the authors were visiting at several universities. Namely, the first named author visited University of Alberta at Edmonton in April 2013 and the second and the fourth named author visited University Paris-Est in June 2013 and in June 2014. The authors would like to thank these universities for their support and hospitality.

\section{Norms of submatrices}

We start with a few general preliminaries and notations. We denote by $B_{2}^{n}$ and $S^{n-1}$ the standard unit Euclidean ball and the unit sphere in $\mathbb{R}^{n}$ and by $|\cdot|$ and $\langle\cdot, \cdot\rangle$ the corresponding Euclidean norm and inner product. Given a set $E \subset\{1, \ldots, N\},|E|$ denotes its cardinality and $B_{2}^{E}$ denotes the unit Euclidean ball in $\mathbb{R}^{E}$, with the convention $B_{2}^{\emptyset}=\{0\}$. 
A standard volume argument implies that for every integer $n$ and for every $\varepsilon \in(0,1)$ there exists an $\varepsilon$-net $\Lambda \subset B_{2}^{n}$ of $B_{2}^{n}$ of cardinality not exceeding $(1+2 / \varepsilon)^{n}$; that is, for every $x \in B_{2}^{n}, \min _{y \in \Lambda}|x-y|<\varepsilon$. In particular, if $\varepsilon \leq 1 / 2$ then the cardinality of $\Lambda$ is not larger than $(2.5 / \varepsilon)^{n}$.

By $\mathcal{M}$ we denote the class of increasing functions $\phi:[0, \infty) \rightarrow[0, \infty)$ such that the function $\ln \phi(1 / \sqrt{x})$ is convex on $(0, \infty)$. The examples of such functions considered in this paper are $\phi(x)=x^{p}$ for some $p>0$ and $\phi(x)=(1 / 2) \exp \left(x^{\alpha}\right)$ for some $\alpha>0$.

Recall that the hypothesis $\mathbf{H}(\phi)$ has been defined in the introduction by (1). Note that this hypothesis is satisfied if

$$
\sup _{a \in S^{n-1}} \mathbb{E} \phi(|\langle X, a\rangle|) \leq \tau .
$$

For $k \leq N$ and random vectors $X_{1}, \ldots, X_{N}$ in $\mathbb{R}^{n}$ we define $A_{k}$ and $B_{k}$ by

$$
A_{k}:=\sup _{\substack{a \in N^{N-1} \\ \operatorname{supp}(a) \leq k}}\left|\sum_{i=1}^{N} a_{i} X_{i}\right|, \quad B_{k}^{2}:=\left.\sup _{\substack{a \in S^{N-1} \\|\operatorname{supp}(a)| \leq k}}|| \sum_{i=1}^{N} a_{i} X_{i}\right|^{2}-\sum_{i=1}^{N} a_{i}^{2}\left|X_{i}\right|^{2} \mid .
$$

We would like to note that $A_{k}$ is the supremum of norms of submatrices consisting of $k$ columns of $A$, while $B_{k}$ plays a crucial role for RIP estimates. We provide more details on the role of $A_{k}$ and $B_{k}$ in the next section.

Recall also a notation from the introduction

$$
M=\max _{i \leq N}\left|X_{i}\right| .
$$

We formulate now the main technical result, Theorem 2.1, which is the key result for both bounds for $A_{k}$ and for $B_{k}$. The role of $A_{k}$ and $B_{k}$ in RIP estimates will be explained in the next section. We postpone the proof to Section 5 ,

Theorem 2.1 Let $p>4, \sigma \in(2, p / 2), \alpha \in(0,2], t>0$, and $\tau, \lambda \geq 1$. Let $X_{1}, \ldots, X_{N}$ be independent random vectors in $\mathbb{R}^{n}$ satisfying hypothesis $\mathbf{H}(\phi)$ with parameter $\tau$ either for $\phi(x)=x^{p}$ or for $\phi(x)=(1 / 2) \exp \left(x^{\alpha}\right)$. For $k \leq N$ define $M_{1}, \beta$ and $C_{\phi}$ in two following cases.

Case 1. $\phi(x)=x^{p}$. We assume that $\lambda \leq p$ and we let $C_{\phi}=e^{4}$,

$M_{1}:=C_{1}(\sigma, \lambda, p) \sqrt{k}\left(\frac{N \tau}{k}\right)^{\sigma / p} \quad$ and $\quad \beta:=C_{2}(\sigma, \lambda)(\tau N)^{-\lambda}+C_{3}(\sigma, \lambda, p) \frac{N^{2} \tau}{t^{p}}$, 
where

$$
\begin{aligned}
& C_{1}(\sigma, \lambda, p)=32 e^{4} \sqrt{\frac{\sigma+\lambda}{1+\lambda / 2}}\left(\frac{2 p}{p-2 \sigma}\right)^{1+2 \sigma / p}\left(\frac{\sigma+\lambda}{\sigma-2}\right)^{2 \sigma / p}(20 e)^{\sigma / p}, \\
& C_{2}(\sigma, \lambda):=\left(\frac{2(\sigma+\lambda)}{5 e(\sigma-2)}\right)^{\lambda} \frac{1}{2 \lambda-1} \quad \text { and } \quad C_{3}(\sigma, \lambda, p):=\frac{(\sigma+\lambda)^{p}}{4(2(\sigma-2))^{p}} .
\end{aligned}
$$

Case 2. $\phi(x)=(1 / 2) \exp \left(x^{\alpha}\right)$. We assume that $\lambda \geq 2$ and we let $C_{\phi}=$ $C^{1 / \alpha}$, where $C$ is an absolute positive constant,

$$
M_{1}:=(C \lambda)^{1 / \alpha} \sqrt{k}\left(\ln \frac{2 N \tau}{k}+\frac{1}{\alpha}\right)^{1 / \alpha}
$$

and

$$
\beta:=\frac{1}{(10 N \tau)^{\lambda}} \exp \left(-\frac{\lambda k^{\alpha / 2}}{(3.5 \ln (2 k))^{2 \alpha}}\right)+\frac{N^{2} \tau}{2 \exp \left((2 t)^{\alpha}\right)} .
$$

In both cases we also assume that $\beta<1 / 32$. Then with probability at least $1-\sqrt{\beta}$ one has

$$
A_{k} \leq(1-4 \sqrt{\beta})^{-1}\left(M+2 \sqrt{C_{\phi} t M}+M_{1}\right)
$$

and

$$
B_{k}^{2} \leq(1-4 \sqrt{\beta})^{-2}\left(4 \sqrt{\beta} M^{2}+\left(8 C_{\phi} t+M_{1}\right) M+2 M_{1}^{2}\right)
$$

We would like to emphasize that $A_{k}$ and $B_{k}$ are of different nature. In particular, Theorem 2.1 in the case $\phi(x)=x^{p}$ has to be applied with different choices of the parameter $\sigma$. We summarize those choices in the following remark.

Remark. In the case $\phi(x)=x^{p}$ we will use the following two choices for $\sigma$ :

1. Choosing $\sigma=p / 4$ and assuming $p>8$ we get

$$
M_{1} \leq C \sqrt{\frac{p}{\lambda}} \sqrt{\frac{p}{p-8}} \sqrt{k}\left(\frac{N \tau}{k}\right)^{1 / 4}
$$

and

$$
\beta \leq\left(\frac{2(p+4 \lambda)}{5 e N \tau(p-8)}\right)^{\lambda} \frac{1}{2 \lambda-1}+\frac{N^{2} \tau(p+4 \lambda)^{p}}{4(2 t(p-8))^{p}}
$$


2. Choosing $\sigma=2+\varepsilon$ with $\varepsilon \leq \min \{1,(p-4) / 4\}$, we get

$$
M_{1} \leq C\left(\frac{p}{p-4}\right)^{1+(4+2 \varepsilon) / p}\left(\frac{\lambda}{\varepsilon}\right)^{2(2+\varepsilon) / p} \sqrt{k}\left(\frac{N \tau}{k}\right)^{(2+\varepsilon) / p}
$$

and

$$
\beta=\left(\frac{2(3+\lambda)}{5 e \varepsilon N \tau}\right)^{\lambda} \frac{1}{2 \lambda-1}+\frac{N^{2} \tau(3+\lambda)^{p}}{4(2 \varepsilon t)^{p}} .
$$

\section{Remarks on optimality.}

1. The case $\phi(x)=x^{p}, p>4$. Let $\tau \geq 1, N \geq\left(64 C_{2}(\sigma, \lambda)\right)^{1 / \lambda}$ and $t=\left(64 N^{2} C_{3}(\sigma, \lambda, p)\right)^{1 / p}$. Then $\beta \leq 1 / 32$ and $\sqrt{t M} \leq C_{4}(\sigma, \lambda, p)\left(M+M_{1}\right)$. Hence with probability larger than $3 / 4$ we have

$$
A_{k} \leq C(\sigma, \lambda, p)\left(M+\sqrt{k}(N \tau / k)^{\sigma / p}\right) .
$$

In Proposition 6.5 below we show that there exist independent random vectors $X_{i}$ 's satisfying the conditions of Theorem 2.1 with $\tau=1$ and such that

$$
A_{k} \geq C(p) \sqrt{k}(N / k)^{1 / p}(\ln (2 N / k))^{-1 / p}
$$

with probability at least $1 / 2$. Note that $M=A_{1} \leq A_{k}$. Therefore

$$
\max \left\{M, C(p) \sqrt{k}(N / k)^{1 / p}(\ln (2 N / k))^{-1 / p}\right\} \leq A_{k} \leq C(\sigma, \lambda, p)\left(M+\sqrt{k}(N / k)^{\sigma / p}\right)
$$

with probability at least $1 / 4$.

2. The case $\phi(x)=(1 / 2) \exp \left(x^{\alpha}\right), \alpha \in[1,2]$. Let $\lambda=2$ and $t=(\ln N)^{1 / \alpha}$. Then $\beta \leq 1 / 32$. Hence with probability larger than $3 / 4$ we have

$$
A_{k} \leq C\left(M+C^{1 / \alpha} \sqrt{k}\left(\ln \left(6^{1 / \alpha} \tau N / k\right)\right)^{1 / \alpha}\right) .
$$

In Proposition 6.7 below we show that there exist independent random vectors $X_{i}$ 's satisfying the conditions of Theorem 2.1 with $\tau$ bounded by an absolute constant and such that

$$
A_{k} \geq \sqrt{k / 2}(\ln (N /(k+1)))^{1 / \alpha}
$$

with probability at least $1 / 2$. Using again that $M=A_{1} \leq A_{k}$ we observe

$$
\max \left\{M, \sqrt{k / 2}(\ln (N /(k+1)))^{1 / \alpha}\right\} \leq A_{k} \leq C\left(M+C^{1 / \alpha} \sqrt{k}\left(\ln \left(6^{1 / \alpha} N / k\right)\right)^{1 / \alpha}\right)
$$

with probability at least $1 / 4$. 


\section{Restricted Isometry Property}

We need more definitions and notations.

Let $T$ be an $n \times N$ matrix and let $1 \leq m \leq N$. The $m$-th isometry constant of $T$ is defined as the smallest number $\delta_{m}=\delta_{m}(T)$ so that

$$
\left(1-\delta_{m}\right)|z|^{2} \leq|T z|^{2} \leq\left(1+\delta_{m}\right)|z|^{2}
$$

holds for all vectors $z \in \mathbb{R}^{N}$ with $|\operatorname{supp}(z)| \leq m$. For $m=0$, we put $\delta_{0}(T)=$ 0 . Let $\delta \in(0,1)$. The matrix $T$ is said to satisfy the Restricted Isometry Property of order $m$ with parameter $\delta$, in short $\operatorname{RIP}_{m}(\delta)$, if $0 \leq \delta_{m}(T) \leq \delta$.

Recall that a vector $z \in \mathbb{R}^{N}$ is called $m$-sparse if $|\operatorname{supp}(z)| \leq m$. The subset of $m$-sparse unit vectors in $\mathbb{R}^{N}$ is denoted by

$$
U_{m}=U_{m}\left(\mathbb{R}^{N}\right):=\left\{z \in \mathbb{R}^{N}:|z|=1,|\operatorname{supp}(z)| \leq m\right\} .
$$

Let $X_{1}, \ldots, X_{N}$ be random vectors in $\mathbb{R}^{n}$ and let $A$ be the $n \times N$ matrix whose columns are the $X_{i}$ 's. By the definition of $B_{m}$ (see (6) ) we clearly have

$$
\begin{aligned}
\max _{i \leq N}\left|\frac{\left|X_{i}\right|^{2}}{n}-1\right|= & \delta_{1}\left(\frac{A}{\sqrt{n}}\right) \leq \delta_{m}\left(\frac{A}{\sqrt{n}}\right)=\sup _{z \in U_{m}}\left|\frac{|A z|^{2}}{n}-1\right| \\
& \leq \frac{B_{m}^{2}}{n}+\max _{i \leq N}\left|\frac{\left|X_{i}\right|^{2}}{n}-1\right| .
\end{aligned}
$$

Thus, in order to have a good bound on $\delta_{m}(A / \sqrt{n})$ we require a strong concentration of each $\left|X_{i}\right|$ around $\sqrt{n}$ and we need to estimate $B_{m}$.

To control the concentration of $\left|X_{i}\right|$ we consider the function $P(\theta)$, defined in the introduction by (2). Note that this function estimates the concentration of the maximum. Therefore, when it is small, we have much better concentration of each $\left|X_{i}\right|$ around $\sqrt{n}$.

We are now ready to state the main result about RIP. Theorem 1.1, announced in the introduction, is a very simplified form of it.

Theorem 3.1 Let $p>4, \alpha \in(0,2], \tau \geq 1$ and $1 \leq n \leq N$. Let $X_{1}, \ldots, X_{N}$ be independent random vectors in $\mathbb{R}^{n}$ satisfying hypothesis $\mathbf{H}(\phi)$ with the parameter $\tau$ either for $\phi(x)=x^{p}$ or for $\phi(x)=(1 / 2) \exp \left(x^{\alpha}\right)$. Let $P(\cdot)$ be as in (2) and $\theta \in(0,1)$.

Case 1. $\phi(x)=x^{p}$. Let $\varepsilon \leq \min \{1,(p-4) / 4\}$. Assume that

$$
\frac{2^{8}}{\varepsilon \theta \tau} \leq N \leq c \theta(c \varepsilon \theta)^{p / 2} n^{p / 4} \sqrt{\tau}
$$


and set

$m=\left[C(\theta, \varepsilon, p) n\left(\frac{N \tau}{n}\right)^{-2(2+\varepsilon) /(p-4-2 \varepsilon)}\right] \quad$ and $\beta=\frac{4}{3 e^{2} \varepsilon^{2} N^{2} \tau^{2}}+\frac{5^{p} N^{2} \tau}{4(2 c \varepsilon \theta)^{p} n^{p / 2}}$,

where

$$
C(\theta, \varepsilon, p)=c\left(\frac{p-4}{p}\right)^{2(p+4+2 \varepsilon) /(p-4-2 \varepsilon)} \varepsilon^{4(2+\varepsilon) /(p-4-2 \varepsilon)} \theta^{2 p /(p-4-2 \varepsilon)},
$$

$c$ and $C$ are absolute positive constants.

Case 2. $\phi(x)=(1 / 2) \exp \left(x^{\alpha}\right)$. Assume that

$$
\frac{1}{\tau} \max \left\{2^{1 / \alpha}, 4 / \theta\right\} \leq N \leq c \theta \sqrt{\tau} \exp \left((1 / 2)(c \theta \sqrt{n})^{\alpha}\right)
$$

and set

$$
m=\left[C^{-2 / \alpha} \theta^{2} n\left(\ln \left(C^{2 / \alpha} N \tau /\left(\theta^{2} n\right)\right)\right)^{-2 / \alpha}\right]
$$

and

$$
\beta=\frac{1}{(10 N \tau)^{2}} \exp \left(\frac{-2 m^{\alpha / 2}}{(3.5 \ln (2 m))^{2 \alpha}}\right)+\frac{N^{2} \tau}{2} \exp \left(-c(\theta \sqrt{n})^{\alpha}\right)
$$

where $c$ and $C$ are absolute positive constants.

Then in both cases we have

$$
\mathbb{P}\left(\delta_{m}(A / \sqrt{n}) \leq \theta\right) \geq 1-\sqrt{\beta}-P(\theta / 2) .
$$

Remarks. 1. Note that for instance in case 1 , the constraint $N \leq c(\theta, \varepsilon, \tau, p) n^{p / 4}$ is not important because for $N \gg n^{p / 4}$ one has

$$
m=\left[C(\theta, \varepsilon, p) n\left(\frac{N \tau}{n}\right)^{-2(2+\varepsilon) /(p-4-2 \varepsilon)}\right]=0
$$

A similar remark is valid in the second case.

2. In most applications $P(\theta) \rightarrow 0$ very fast as $n, N \rightarrow \infty$. For example, for so-called isotropic log-concave random vectors it follows from results of Paouris ([27, 28], see also [18, 16] or Lemma 3.3 of [4]). As another example consider the model when $X_{i}$ 's are i.i.d. and moreover the coordinates of $X_{1}$ are i.i.d. random variables distributed as a random variable $\xi$. In the 
case when $\xi$ is of variance one and has finite $p$-th moment, $p>4$, then by Rosenthal's inequality $P(\theta)$ is well bounded (for a precise bound see Corollary 6.4 below, see also Proposition 1.3 of [31]). Another case is when $\xi$ is the Weibull random variable of variance one, that is consider $\xi_{0}$ such that $\mathbb{P}\left(\left|\xi_{0}\right|>t\right)=\exp \left(-t^{\alpha}\right)$ for $\alpha \in(0,2]$ and let $\xi=\xi_{0} / \sqrt{\mathbb{E} \xi_{0}^{2}}$. By Lemma 3.4 from [4] (see also Theorem 1.2.8 in [11]), $P(\theta)$ satisfies (37) below.

3. Optimality. Taking $\varepsilon$ in the Case 1 of order $(p-4)^{2} / \ln (2 N \tau / n)$ and assuming that it satisfies the condition of the theorem, we observe that in Case 1

$$
m=\left[C(\theta, p) n\left(\frac{N \tau}{n}\right)^{-4 /(p-4)}\left(\ln \frac{2 N \tau}{n}\right)^{-8 /(p-4)}\right] .
$$

Moreover, Proposition 6.6 below shows that for $q>p>4$ there are independent random vectors $X_{i}$ 's satisfying hypothesis $\mathbf{H}(\phi)$ with parameter $\tau=\tau(p, q)$ and such that for $\theta=1 / 2, N \leq C(p, q) n^{p / 4}(\ln (2 N / n))^{-p / 2}$ one can't get better estimate than

$$
m \leq 8(N / n)^{-2 /(q-2)} n .
$$

4. Optimality. In Case 2 with $\tau$ bounded by an absolute constant and $\alpha \in$ $[1,2]$, let $c_{0} n \leq N \leq c_{1} \exp \left(c_{2} n^{\alpha / 2}\right), \theta=0.4$ and assume that $P(\theta / 2)$ is small enough. Then $\mathbb{P}\left(\delta_{m} \leq 1 / 2\right) \geq 1 / 2$ provided that $m=n\left(C \ln \left(C^{2 / \alpha} N / n\right)\right)^{2 / \alpha}$. Proposition 6.7 below shows that the estimate for $m$ is sharp, that is in general $m$ can't be larger than $m=n(C \ln (2 N / n))^{2 / \alpha}$.

Proof. We first pass to the subset $\Omega_{0}$ of our initial probability space where

$$
\max _{i \leq N}\left|\frac{\left|X_{i}\right|^{2}}{n}-1\right| \leq \theta / 2
$$

Note that by (2) the probability of this event is at least $1-P(\theta / 2)$ and if this event occurs then we also have

$$
\max _{i \leq N}\left|X_{i}\right| \leq 3 \sqrt{n} / 2
$$

We will apply Theorem 2.1 with $k=m, t=\theta \sqrt{n} /\left(100 C_{\phi}\right)$, where $C_{\phi}$ is the constant from Theorem 2.1. Additionally we assume that $\beta \leq 2^{-9} \theta^{2}$ and $M_{1} \leq t$. Then with probability at least $1-\sqrt{\beta}-P(\theta / 2)$ we have

$$
B_{m}^{2} \leq(16 \sqrt{\beta}+\theta / 4) n \leq \theta n / 2 .
$$


Together with (10) this proves $\delta_{m}(A / \sqrt{n}) \leq \theta$. Thus we only need to check when the estimates for $\beta$ and $M_{1}$ are satisfied.

Case 1. $\phi(x)=x^{p}$. We start by proving the estimate for $M_{1}$. We let $\sigma=2+\varepsilon, \varepsilon \leq \min \{1,(p-4) / 4\}$ and $\lambda=2$. Then by Theorem 2.1 (see also the Remark following it), for some absolute constant $C$ we have

$$
M_{1} \leq C\left(\frac{p}{p-4}\right)^{1+(4+2 \varepsilon) / p}\left(\frac{1}{\varepsilon}\right)^{2(2+\varepsilon) / p} \sqrt{m}\left(\frac{N \tau}{m}\right)^{(2+\varepsilon) / p} .
$$

Therefore the estimate $M_{1} \leq c \theta \sqrt{n}$ with $c=1 /\left(100 e^{4}\right)$ is satisfied provided that

$$
m=\left[C(\theta, \varepsilon, p) n\left(\frac{N \tau}{n}\right)^{-2(2+\varepsilon) /(p-4-2 \varepsilon)}\right],
$$

with $C(\theta, \varepsilon, p)$ defined in (11) and the absolute constants properly adjusted.

Now we estimate the probability. From Theorem 2.1 (and the Remark following it), with our choice of $t$ and $\lambda$ we have

$$
\beta \leq \frac{4}{3 e^{2} \varepsilon^{2} N^{2} \tau^{2}}+\frac{5^{p} N^{2} \tau}{4(2 c \varepsilon \theta)^{p} n^{p / 2}} \leq 2^{-9} \theta^{2}
$$

provided that $2^{8} /(\varepsilon \theta \tau) \leq N \leq 2^{-4} \theta \sqrt{\tau}(0.4 c \varepsilon \theta)^{p / 2} n^{p / 4}$. This completes the proof of the first case.

Case 2. $\phi(x)=(1 / 2) \exp \left(x^{\alpha}\right)$. As in the first case we start with the condition $M_{1} \leq t$. We choose $\lambda=4$. Note that $N \tau / m \geq 2^{1 / \alpha}$ as $N \tau \geq 2^{1 / \alpha} n$. Therefore for some absolute constant $C$,

$$
M_{1} \leq \sqrt{m}(C \ln (2 N \tau / m))^{1 / \alpha} .
$$

Therefore the condition $M_{1} \leq t$ is satisfied provided that

$$
m \leq C_{1}^{-2 / \alpha} \theta^{2} n\left(\ln \left(C_{1}^{2 / \alpha} N \tau /\left(\theta^{2} n\right)\right)\right)^{-2 / \alpha}
$$

for an absolute positive constant $C_{1}$. This justifies the choice of $m$.

Now we estimate the probability. From Theorem 2.1 with our choice of $t$ and $\lambda$ we have

$$
\beta \leq \frac{1}{(10 N \tau)^{2}} \exp \left(\frac{-2 m^{\alpha / 2}}{(3.5 \ln (2 m))^{2 \alpha}}\right)+\frac{N^{2} \tau}{2} \exp \left(-c(\theta \sqrt{n})^{\alpha}\right) \leq 2^{-9} \theta^{2},
$$

provided that $4 /(\theta \tau) \leq N \leq 2^{-5} \theta \sqrt{\tau} \exp \left(c(\theta \sqrt{n})^{\alpha}\right)$. This completes the proof. 


\section{Approximating the covariance matrix}

We start with the following $\varepsilon$-net argument for bilinear forms, which will be used below.

Lemma 4.1 Let $m \geq 1$ be an integer and $T$ be an $m \times m$ matrix. Let $\varepsilon \in(0,1 / 2)$ and $\mathcal{N} \subset B_{2}^{m}$ be an $\varepsilon$-net of $B_{2}^{m}$ (in the Euclidean metric). Then

$$
\sup _{x \in B_{2}^{m}}|\langle T x, x\rangle| \leq(1-2 \varepsilon)^{-1} \sup _{y \in \mathcal{N}}|\langle T y, y\rangle| .
$$

Proof. Let $S=T+T^{*}$. For any $x, y \in \mathbb{R}^{m}$,

$$
\langle S x, x\rangle=\langle S y, y\rangle+\langle S x, x-y\rangle+\langle S(x-y), y\rangle .
$$

Therefore $|\langle S x, x\rangle| \leq|\langle S y, y\rangle|+2|x-y|\|S\|$. Since $S$ is symmetric, we have

$$
\|S\|=\sup _{x \in B_{2}^{m}}|\langle S x, x\rangle| .
$$

Thus, if $|x-y| \leq \varepsilon$, then

$$
\|S\| \leq \sup _{y \in \mathcal{N}}|\langle S y, y\rangle|+2 \varepsilon\|S\|
$$

and

$$
\sup _{x \in B_{2}^{m}}|\langle S x, x\rangle| \leq(1-2 \varepsilon)^{-1} \sup _{y \in \mathcal{N}}|\langle S y, y\rangle| .
$$

Since $T$ is a real matrix, then for every $x \in \mathbb{R}^{m},\langle S x, x\rangle=2\langle T x, x\rangle$. This concludes the proof.

Now we can prove the following technical lemma, which emphasizes the role of the parameter $A_{k}$ in estimates of the distance between the covariance matrix and the empirical one. This role was first recognized in [8] and [2]. Other versions of the lemma appeared in [4, 5]. Its proof uses the symmetrization method as in [25].

Lemma 4.2 Let $\tau \geq 1,1 \leq k<N$ and $X_{1}, \ldots, X_{N}$ be independent random vectors in $\mathbb{R}^{n}$. Let $p \geq 2, \alpha \in(0,2]$. Let $\phi$ be either $\phi(t)=t^{p}$ in which case we set $C_{\phi}=8 \tau^{2 / p} N^{2 / \min (p, 4)}$ and assume

$$
\forall 1 \leq i \leq N \quad \forall a \in S^{n-1} \quad \mathbb{E}\left|\left\langle X_{i}, a\right\rangle\right|^{p} \leq \tau,
$$


or $\phi(t)=(1 / 2) \exp \left(t^{\alpha}\right)$ in which case we assume that $X_{i}$ 's satisfy hypothesis $\mathbf{H}(\phi)$ with parameter $\tau$ and set $C_{\phi}=8 \sqrt{C_{\alpha} N \tau}$, where $C_{\alpha}=(8 / \alpha) \Gamma(4 / \alpha)$, $\Gamma(\cdot)$ is the Gamma function. Then, for every $A, Z>0$,

$$
\sup _{a \in S^{n-1}}\left|\sum_{i=1}^{N}\left(\left\langle X_{i}, a\right\rangle^{2}-\mathbb{E}\left\langle X_{i}, a\right\rangle^{2}\right)\right| \leq 2 A^{2}+6 \sqrt{n} Z+C_{\phi}
$$

with probability larger than

$$
1-4 \exp (-n)-4 \mathbb{P}\left(A_{k}>A\right)-4 \times 9^{n} \sup _{a \in S^{n-1}} \mathbb{P}\left(\left(\sum_{i>k}\left(\left\langle X_{i}, a\right\rangle^{*}\right)^{4}\right)^{1 / 2}>Z\right) .
$$

The term involving $Z$ in the upper bound will be bounded later using general estimates in Lemma 4.4. Thus Lemma 4.2 clearly stresses the fact that in order to estimate the distance between the covariance matrix and the empirical one, it will remain to estimate $A_{k}$, to get $A$.

Proof: Let $\Lambda \subset \mathbb{R}^{n}$ be a (1/4)-net of the unit Euclidean ball in the Euclidean metric of cardinality not greater than $9^{n}$. Let $\left(\varepsilon_{i}\right)_{1 \leq i \leq N}$ be i.i.d. \pm 1 Bernoulli random variables of parameter $1 / 2$. By Hoeffding's inequality, for every $t>0$ and every $\left(s_{i}\right)_{1 \leq i \leq N} \in \mathbb{R}^{N}$,

$$
\mathbb{P}_{\left(\varepsilon_{i}\right)}\left(\left|\sum_{i=1}^{N} \varepsilon_{i} s_{i}\right| \geq t\left(\sum_{i=1}^{N}\left|s_{i}\right|^{2}\right)^{1 / 2}\right) \leq 2 \exp \left(-t^{2} / 2\right) .
$$

Fix an arbitrary $1 \leq k<N$. For every $\left(s_{i}\right)_{1 \leq i \leq N} \in \mathbb{R}_{+}^{N}$ there exists a permutation $\pi$ of $\{1, \ldots, N\}$ such that

$$
\left|\sum_{i=1}^{N} \varepsilon_{i} s_{i}\right| \leq \sum_{i=1}^{k} s_{i}^{*}+\left|\sum_{i=k+1}^{N} \varepsilon_{\pi(i)} s_{i}^{*}\right|
$$

where $\left(s_{i}^{*}\right)_{i}$ denotes a non-increasing rearrangement of $\left(\left|s_{i}\right|\right)_{i}$.

Also, it is easy to check using (6) that for any $a \in S^{n-1}$ and any $I \subset$ $\{1, \ldots, N\}$ with $|I| \leq k, \sum_{i \in I}\left\langle X_{i}, a\right\rangle^{2} \leq A_{k}^{2}$.

Thus, for every $a \in S^{n-1}$,

$$
\mathbb{P}_{\left(\varepsilon_{i}\right)}\left(\left|\sum_{i=1}^{N} \varepsilon_{i}\left\langle X_{i}, a\right\rangle^{2}\right| \leq A_{k}^{2}+t\left(\sum_{i=k+1}^{N}\left(\left\langle X_{i}, a\right\rangle^{*}\right)^{4}\right)^{1 / 2}\right) \geq 1-2 \exp \left(-t^{2} / 2\right) .
$$


Note that $\sum_{i=1}^{N} \varepsilon_{i}\left\langle X_{i}, a\right\rangle^{2}=\sum_{i \in E}\left\langle X_{i}, a\right\rangle^{2}-\sum_{i \in E^{c}}^{N}\left\langle X_{i}, a\right\rangle^{2}$ for some set $E \subset$ $\{1, \ldots, N\}$ and we can apply a union bound argument indexed by $\Lambda$ together with Lemma 4.1. We get that

$$
\begin{gathered}
\mathbb{P}_{\left(\varepsilon_{i}\right)}\left(\sup _{a \in S^{n-1}}\left|\sum_{i=1}^{N} \varepsilon_{i}\left\langle X_{i}, a\right\rangle^{2}\right| \leq 2\left[A_{k}^{2}+t \sup _{a \in \Lambda}\left(\sum_{i=k+1}^{N}\left(\left\langle X_{i}, a\right\rangle^{*}\right)^{4}\right)^{1 / 2}\right]\right) \\
\geq 1-2 \times 9^{n} \exp \left(-t^{2} / 2\right) .
\end{gathered}
$$

Using again a union bound argument and the triangle inequality to estimate the probability that the $\left(X_{i}\right)$ satisfy

$$
\sup _{a \in \Lambda}\left(\sum_{i=k+1}^{N}\left(\left\langle X_{i}, a\right\rangle^{*}\right)^{4}\right)^{1 / 2}>Z,
$$

and choosing $t=3 \sqrt{n}$ (so that $2 \cdot 9^{n} \exp \left(-t^{2} / 2\right) \leq e^{-n}$ ) we get that

$$
\sup _{a \in S^{n-1}}\left|\sum_{i=1}^{N} \varepsilon_{i}\left\langle X_{i}, a\right\rangle^{2}\right| \leq 2 A^{2}+6 \sqrt{n} Z
$$

with probability larger than

$$
1-e^{-n}-\mathbb{P}\left(A_{k}>A\right)-9^{n} \sup _{a \in S^{n-1}} \mathbb{P}\left(\left(\sum_{i=k+1}^{N}\left(\left\langle X_{i}, a\right\rangle^{*}\right)^{4}\right)^{1 / 2}>Z\right) .
$$

Now we transfer the result from Bernoulli random variables to centered random variables (see [21], Section 6.1). By the triangle inequality, for every $s, t>0$, one has

$$
\begin{gathered}
m(s) \mathbb{P}\left(\sup _{a \in S^{n-1}}\left|\sum_{i=1}^{N}\left(\left\langle X_{i}, a\right\rangle^{2}-\mathbb{E}\left\langle X_{i}, a\right\rangle^{2}\right)\right|>s+t\right) \\
\leq 2 \mathbb{P}\left(\sup _{a \in S^{n-1}}\left|\sum_{i=1}^{N} \varepsilon_{i}\left\langle X_{i}, a\right\rangle^{2}\right|>t\right)
\end{gathered}
$$

where $m(s)=\inf _{a \in S^{n-1}} \mathbb{P}\left(\left|\sum_{i=1}^{N}\left(\left\langle X_{i}, a\right\rangle^{2}-\mathbb{E}\left\langle X_{i}, a\right\rangle^{2}\right)\right| \leq s\right)$.

To conclude the proof it is enough to find $s$ so that $m(s) \geq 1 / 2$. To this end we will use a general Lemma 4.3 (below). First consider $\phi(t)=t^{p}$. 
For $a \in S^{n-1}$, set $Z_{i}=\left|\left\langle X_{i}, a\right\rangle\right|^{2} / \tau^{2 / p}$ and $q=p / 2$. Then by Lemma 4.3 we have $m(s) \geq 1 / 2$ for $s=4 \tau^{2 / p} N^{1 / r}$ and $r=\min (p / 2,2)$. Now consider $\phi(t)=(1 / 2) \exp \left(t^{\alpha}\right)$. Then for every $a \in S^{n-1}$ and every $i \leq N$ using hypothesis $\mathbf{H}(\phi)$ we have

$$
\mathbb{E}\left|\left\langle X_{i}, a\right\rangle\right|^{4} \leq 8 \tau \int_{0}^{\infty} t^{3} \exp \left(-t^{\alpha}\right) d t=\frac{8 \tau}{\alpha} \Gamma\left(\frac{4}{\alpha}\right):=\tau C_{\alpha}
$$

Given $a \in S^{n-1}$, set $Z_{i}=\left|\left\langle X_{i}, a\right\rangle\right|^{2} / \sqrt{\tau C_{\alpha}}$. Then $\mathbb{E} Z_{i}^{2} \leq 1$. Applying again Lemma 4.3 (with $q=2$ ), we observe that $m(s) \geq 1 / 2$ for $s=4 \sqrt{C_{\alpha} N}$. This completes the proof.

It remains to prove the following general lemma. For convenience of the argument above, we formulate this lemma using two powers $q$ and $r$ rather than just one.

Lemma 4.3 Let $q \geq 1$ and $Z_{1}, \ldots, Z_{N}$ be independent non-negative random variables satisfying

$$
\forall 1 \leq i \leq N \quad \mathbb{E} Z_{i}^{q} \leq 1
$$

Let $r=\min (q, 2)$, then

$$
\forall z \geq 4 N^{1 / r} \quad \mathbb{P}\left(\left|\sum_{i=1}^{N}\left(Z_{i}-\mathbb{E} Z_{i}\right)\right| \leq z\right) \geq \frac{1}{2} .
$$

Proof: By definition of $r$, we have for all $i=1, \ldots, N, \mathbb{E} Z_{i}^{r} \leq 1$. Since the $Z_{i}$ 's are independent, we deduce by a classical symmetrization argument that

$\mathbb{E}\left|\sum_{i=1}^{N}\left(Z_{i}-\mathbb{E} Z_{i}\right)\right| \leq 2 \mathbb{E}_{\left(\varepsilon_{i}\right)}\left|\sum_{i=1}^{N} \varepsilon_{i} Z_{i}\right| \leq 2 \mathbb{E}\left(\sum_{i=1}^{N} Z_{i}^{2}\right)^{1 / 2} \leq 2 \mathbb{E}\left(\sum_{i=1}^{N} Z_{i}^{r}\right)^{1 / r}$

since $r \in[1,2]$. From $\mathbb{E} Z_{i}^{r} \leq 1$, we get that

$$
\mathbb{E}\left|\sum_{i=1}^{N}\left(Z_{i}-\mathbb{E} Z_{i}\right)\right| \leq 2 \mathbb{E}\left(\sum_{i=1}^{N} Z_{i}^{r}\right)^{1 / r} \leq 2\left(\sum_{i=1}^{N} \mathbb{E} Z_{i}^{r}\right)^{1 / r} \leq 2 N^{1 / r}
$$

By Markov's inequality we get

$$
\mathbb{P}\left(\left|\sum_{i=1}^{N}\left(Z_{i}-\mathbb{E} Z_{i}\right)\right| \geq 4 N^{1 / r}\right) \leq \frac{1}{2}
$$


and since $z \geq 4 N^{1 / r}$, this implies the required estimate.

The following lemma is standard (cf. Lemma 5.8 in [21], which however contains a misprint).

Lemma 4.4 Let $q>0$ and let $Z_{1}, \ldots, Z_{N}$ be independent non-negative random variables satisfying

$$
\forall 1 \leq i \leq N \quad \forall t \geq 1 \quad \mathbb{P}\left(Z_{i} \geq t\right) \leq 1 / t^{q} .
$$

Then, for every $s>1$, with probability larger than $1-s^{-k}$, one has

$$
\sum_{i=k}^{N} Z_{i}^{*} \leq \begin{cases}\frac{(2 e s)^{1 / q}}{1-q} N^{1 / q} k^{1-1 / q} & \text { if } 0<q<1 \\ 2 e s N \ln \left(\frac{e N}{k}\right) & \text { if } q=1 \\ \frac{12 q(e s)^{1 / q}}{q-1} N & \text { if } q>1 .\end{cases}
$$

Proof: Assume first that $0<q \leq 1$. It is clear that

$$
\forall 1 \leq i \leq N \quad \mathbb{P}\left(Z_{i}^{*}>t\right) \leq\left(\begin{array}{c}
N \\
i
\end{array}\right) t^{-i q} \leq\left(N e / i t^{q}\right)^{i}
$$

where we used the inequality $\left(\begin{array}{c}N \\ i\end{array}\right) \leq(N e / i)^{i}$. Thus if $e N t^{-q} \leq 1$, then

$$
\mathbb{P}\left(\sup _{i \geq k} i^{1 / q} Z_{i}^{*}>t\right) \leq \sum_{i \geq k}\left(N e / t^{q}\right)^{i}=\left(\frac{e N}{t^{q}}\right)^{k}\left(1-e N t^{-q}\right)^{-1} .
$$

Therefore if $e N t^{-q} \leq 1 / 2$, then $\mathbb{P}\left(\sup _{i \geq k} i^{1 / q} Z_{i}^{*}>t\right) \leq\left(2 e N t^{-q}\right)^{k}$. Since the inequality is trivially true if $e N t^{-q} \geq 1 / 2$, it is proved for every $t>0$. Therefore for $q<1$ we have

$$
\sum_{i=k}^{N} Z_{i}^{*} \leq t \sum_{i=k}^{\infty} i^{-1 / q} \leq t\left(k^{-1 / q}-\frac{k^{1-1 / q}}{1-1 / q}\right) \leq \frac{t}{1-q} k^{1-1 / q}
$$

with probability larger than $1-\left(2 e N / t^{q}\right)^{k}$. Choosing $t=(2 e s N)^{1 / q}$, we obtain the estimate in the case $0<q<1$.

For $q=1$ we have

$$
\sum_{i=k}^{N} Z_{i}^{*} \leq t \sum_{i=k}^{N} i^{-1} \leq t\left(\frac{1}{k}+\ln (N / k)\right) \leq t \ln (e N / k)
$$


with probability larger than $1-(2 e N / t)^{k}$. To obtain the desire estimate choose $t=2 e s N$.

Now assume that $q>1$. Set $\ell=\left\lceil\log _{2} k\right\rceil$. The same computation as before for the scale $\left(2^{i / q}\right)$ instead of $\left(i^{1 / q}\right)$ gives that

$$
\mathbb{P}\left(\sup _{i \geq \ell} 2^{i / q} Z_{2^{i}}^{*}>t\right) \leq \sum_{i \geq \ell}\left(N e t^{-q}\right)^{2^{i}} \leq\left(2 e N t^{-q}\right)^{2^{\ell}} .
$$

Note also that

$$
\mathbb{P}\left(k^{1 / q} Z_{k}^{*}>t\right) \leq\left(N e t^{-q}\right)^{k} .
$$

Thus

$$
\begin{aligned}
\sum_{i=k}^{N} Z_{i}^{*} & \leq k Z_{k}^{*}+\sum_{i=\ell}^{\left\lceil\log _{2} N\right\rceil} 2^{i} Z_{2^{i}}^{*} \leq t\left(k^{1-1 / q}+(4 N)^{1-1 / q} /\left(2^{1-1 / q}-1\right)\right) \\
& \leq t\left(k^{1-1 / q}+\frac{2 q}{1-q}(4 N)^{1-1 / q}\right) \leq t \frac{3 q}{1-q}(4 N)^{1-1 / q}
\end{aligned}
$$

with probability larger than $\left(N e t^{-q}\right)^{k}+\left(2 N e t^{-q}\right)^{k}$. Thus, taking $t=(4 e s N)^{1 / q}$, we obtain

$$
\mathbb{P}\left(\sum_{i=k}^{N} Z_{i}^{*} \leq \frac{12 q(e s)^{1 / q}}{q-1} N\right) \geq 1-s^{-k} .
$$

We are now ready to tackle the problem of approximating the covariance matrix by the empirical covariance matrices, under hypothesis $H(\phi)$ with $\phi(t)=t^{p}$. As our proof works for all $p>4$, we also include the case $p>8$ originally solved in [25] (under additional assumption on $\max _{i}\left|X_{i}\right|$ ). For clarity, we split the result into two theorems. The case $4<p \leq 8$ has been stated as Theorem 1.2 in the Introduction.

Before we state our result, let us remark that $p>2$ is a necessary condition. Indeed, let $\left(e_{i}\right)_{1 \leq i \leq n}$ be an orthonormal basis of $\mathbb{R}^{n}$ and let $Z$ be a random vector such that $Z=\sqrt{n} e_{i}$ with probability $1 / n$. The covariance matrix of $Z$ is the identity $I$. Let $A$ be an $n \times N$ random matrix with independent columns distributed as $Z$. Note that if $\left\|\frac{1}{N} A A^{\top}-I\right\|<1$ with some probability, then $A A^{\top}$ is invertible with the same probability. It is known (coupon collector's problem) that $N \sim n \log n$ is needed to have $\left\{Z_{i}: i \leq N\right\}=\left\{\sqrt{n} e_{i}: i \leq n\right\}$ with probability, say, $1 / 2$. Thus for vector 
$Z$, the hypothesis $H(\phi), \phi(t)=t^{2}$ is satisfied but $N \sim n \log n$ is needed for the covariance matrix to be well approximated by the empirical covariance matrices with probability $1 / 2$.

We also would like to mention that we don't know how sharp the power $\gamma / p$ appearing in the bound below is. In particular, it is not clear if it can be improved to $1 / 2$.

Theorem 4.5 Let $4<p \leq 8$ and $\phi(t)=t^{p}$. Let $X_{1}, \ldots, X_{N}$ be independent random vectors in $\mathbb{R}^{n}$ satisfying hypothesis $\mathbf{H}(\phi)$. Let $\varepsilon \leq \min \{1,(p-4) / 4\}$ and $\gamma=p-4-2 \varepsilon$. Then with probability larger than

$$
1-8 e^{-n}-2 \varepsilon^{-p / 2} \max \left\{N^{-3 / 2}, n^{-(p / 4-1)}\right\}
$$

one has

$\sup _{a \in S^{n-1}}\left|\frac{1}{N} \sum_{i=1}^{N}\left(\left\langle X_{i}, a\right\rangle^{2}-\mathbb{E}\left\langle X_{i}, a\right\rangle^{2}\right)\right| \leq C\left(\frac{1}{N} \max _{i \leq N}\left|X_{i}\right|^{2}+C(p, \varepsilon)\left(\frac{n}{N}\right)^{\gamma / p}\right)$,

where

$$
C(p, \varepsilon)=(p-4)^{-1 / 2} \varepsilon^{-4(2+\varepsilon) / p} .
$$

and $C$ is an absolute constant.

An immediate consequence of this theorem is the following corollary.

Corollary 4.6 Under assumptions of Theorem 4.7, assuming additionally that $\max _{i}\left|X_{i}\right|^{2} \leq C n^{\gamma / p} N^{1-\gamma / p}$ with high probability, we have with high probability

$$
\sup _{a \in S^{n-1}}\left|\frac{1}{N} \sum_{i=1}^{N}\left(\left\langle X_{i}, a\right\rangle^{2}-\mathbb{E}\left\langle X_{i}, a\right\rangle^{2}\right)\right| \leq C_{1} C(p, \varepsilon)\left(\frac{n}{N}\right)^{\gamma / p},
$$

where $C$ and $C_{1}$ are absolute positive constants.

Theorem 4.7 There exists a universal positive constant $C$ such that the following holds. Let $p>8, \alpha \in(0,2]$. Let $\phi$ and $C_{\phi}$ be either $\phi(t)=t^{p}$ and $C_{\phi}=C$ or $\phi(t)=(1 / 2) \exp \left(t^{\alpha}\right)$ and $C_{\phi}=(C / \alpha)^{2.5 / \alpha}$. Let $X_{1}, \ldots, X_{N}$ be independent random vectors in $\mathbb{R}^{n}$ satisfying hypothesis $\mathbf{H}(\phi)$. In the case $\phi(t)=t^{p}$ we also define

$$
p_{0}=8 e^{-n}+2\left(\frac{3 p-8}{6(p-8)}\right)^{p / 2} N^{-(p-8) / 8} n^{-p / 8}
$$


and in the case $\phi(t)=(1 / 2) \exp \left(t^{\alpha}\right)$, we assume $N \geq(4 / \alpha)^{8 / \alpha}$ and define

$$
p_{0}=8 e^{-n}+\frac{1}{(10 N)^{4}} \exp \left(\frac{4 n^{\alpha / 2}}{(3.5 \ln (2 n))^{2 \alpha}}\right)+\frac{N^{2}}{2 \exp \left((2 n N)^{\alpha / 4}\right)} .
$$

Then in both cases with probability larger than $1-p_{0}$ one has

$$
\sup _{a \in S^{n-1}}\left|\frac{1}{N} \sum_{i=1}^{N}\left(\left\langle X_{i}, a\right\rangle^{2}-\mathbb{E}\left\langle X_{i}, a\right\rangle^{2}\right)\right| \leq \frac{C}{N} \max _{i \leq N}\left|X_{i}\right|^{2}+C_{\phi} \sqrt{\frac{n}{N}} .
$$

As our argument works in all cases we prove both theorems together.

Proof of Theorems 4.5 and 4.7. We first consider the case $\phi=t^{p}$. Note that in this case

$$
\mathbb{E}\left|\left\langle X_{i}, a\right\rangle\right|^{4} \leq 1+\int_{1}^{\infty} \mathbb{P}\left(\left|X_{i}\right|^{4}>t\right) d t \leq 1+\int_{1}^{\infty} 4 s^{3-p} d s=\frac{p}{p-4} .
$$

Thus, by Lemma 4.2 it is enough to estimate $A^{2}+\sqrt{n} Z+\sqrt{p /(p-4)} \sqrt{N}$ and the corresponding probabilities. We choose $k=n$.

In the case $\phi=t^{p}$ we apply Lemma 4.4 with $Z_{i}=\left|\left\langle X_{i}, a\right\rangle\right|^{4}, i \leq N$, $q=p / 4>1$ and $s=9 e$. It gives

$$
\mathbb{P}\left(\left(\sum_{i>n}\left(\left\langle X_{i}, a\right\rangle^{*}\right)^{4}\right)^{1 / 2}>Z\right) \leq(9 e)^{-n}
$$

for

$$
Z=\sqrt{\frac{12 q}{q-1}}(e s)^{1 / 2 q} \sqrt{N}=\sqrt{\frac{12 p}{p-4}}(3 e)^{4 / p} \sqrt{N} .
$$

Now we estimate $A_{n}$, using Theorem 2.1.

Case 1: $4<p \leq 8$ (Theorem 4.5). We apply Theorem 2.1 (and the Remark following it), with $\sigma=2+\varepsilon$, where $\varepsilon<(p-4) / 4, \lambda=3$ and $t=3 N^{2 / p} n^{\delta}$ for $\delta=1 / 2-2 / p$. Then

$$
M_{1} \leq C(p, \varepsilon) \sqrt{n}(N / n)^{(2+\varepsilon) / p},
$$

where

$$
C_{0}(p, \varepsilon)=C\left(\frac{1}{p-4}\right)^{(p-4-2 \varepsilon) / p}\left(\frac{1}{\varepsilon}\right)^{2(2+\varepsilon) / p}
$$


and

$$
\beta \leq \frac{1}{5}\left(\frac{12}{5 e \varepsilon N}\right)^{3}+\frac{1}{4(p-4)^{p} n^{\delta p}} \leq \varepsilon^{-p} \max \left\{N^{-3}, n^{-\delta p}\right\} \leq 1 / 64
$$

provided that $n$ is large enough. Then, using $\delta=1 / 2-2 / p$, we obtain

$$
\begin{gathered}
A_{n}^{2} \leq C\left(\max _{i \leq N}\left|X_{i}\right|^{2}+N^{2 / p} n^{\delta} \max _{i \leq N}\left|X_{i}\right|+C_{0}^{2}(p, \varepsilon) n(N / n)^{2(2+\varepsilon) / p}\right) \\
\leq 2 C\left(\max _{i \leq N}\left|X_{i}\right|^{2}+C_{0}^{2}(p, \varepsilon) n(N / n)^{2(2+\varepsilon) / p}\right) .
\end{gathered}
$$

Combining all estimates and noticing that $(p-4)^{-\gamma}<2$, we obtain that the desired estimate holds with probability

$$
1-8 e^{-n}-2 \varepsilon^{-p / 2} \max \left\{N^{-3 / 2}, n^{-(p / 4-1)}\right\} .
$$

Case 2: $p>8$ (Theorem 4.7). In this case we apply Theorem 2.1 (see also the Remark following it), with $\sigma=p / 4, \lambda=(p-4) / 2, t=3(n N)^{1 / 4}$. Then $M_{1} \leq C \sqrt{n}(N / n)^{1 / 4}$ and

$$
\begin{gathered}
\beta \leq\left(\frac{2(3 p-8)}{5 e(p-8) N}\right)^{(p-4) / 2} \frac{1}{p-5}+\frac{(3 p-8)^{p}}{4(6(p-8))^{p} N^{(p-8) / 4} n^{p / 4}} \\
\leq\left(\frac{3 p-8}{6(p-8)}\right)^{p} N^{-(p-8) / 4} n^{-p / 4} \leq 1 / 64,
\end{gathered}
$$

provided that $N$ is large enough. Thus with probability at least $1-\sqrt{\beta}$ we have

$A_{n}^{2} \leq C\left(\max _{i \leq N}\left|X_{i}\right|^{2}+(n N)^{1 / 4} \max _{i \leq N}\left|X_{i}\right|+\sqrt{n N}\right) \leq 2 C\left(\max _{i \leq N}\left|X_{i}\right|^{2}+\sqrt{n N}\right)$.

Combining all estimates we obtain that the desired estimate holds with probability

$$
1-8 e^{-n}-2\left(\frac{3 p-8}{6(p-8)}\right)^{p / 2} N^{-(p-8) / 8} n^{-p / 8}
$$

Case 3: $\phi(t)=(1 / 2) \exp \left(t^{\alpha}\right) \quad$ (Theorem 4.7). As in Case 2 we apply Lemma 4.2. It implies that it is enough to estimate $A^{2}+\sqrt{n} Z+\sqrt{C(\alpha) N}$, 
with $C(\alpha)$ from Lemma 4.2, and the corresponding probabilities. A direct calculations show that in this case we have for $C_{\alpha}^{\prime}=(4 / \alpha)^{1 / \alpha}$ and $t>1$,

$$
\mathbb{P}\left(\left(|X| / C_{\alpha}^{\prime}\right)^{4}>t\right) \leq 2 \exp \left(C_{\alpha}^{\prime}\right) t^{\alpha / 4} \leq \frac{1}{t^{2}} .
$$

We apply Lemma 4.4 with $Z_{i}=\left|\left\langle X_{i}, a\right\rangle\right|^{4} / \sqrt{C_{\alpha}^{\prime}}, i \leq N, q=2$ and $s=9 e$. It gives

$$
\mathbb{P}\left(\left(\sum_{i>k}\left(\left\langle X_{i}, a\right\rangle^{*}\right)^{4}\right)^{1 / 2}>Z\right) \leq(9 e)^{-n}
$$

for

$$
Z=\left(C_{\alpha}^{\prime}\right)^{1 / 4} 6 \sqrt{6} e \sqrt{N} .
$$

To estimate $A_{n}$ we use Theorem 2.1 with $t=(n N)^{1 / 4}$ and

$$
\lambda=10(N / n)^{\alpha / 4} \min \left\{1,(\alpha \ln (2 N / n))^{-1}\right\} .
$$

Note that

$$
\max \left\{4,10(N / n)^{\alpha / 4}(\ln (2 N / n))^{-1}\right\} \leq \lambda \leq 10(N / n)^{\alpha / 4} .
$$

Then for absolute positive constants $C, C^{\prime}$,

$$
M_{1} \leq \sqrt{n}(C \lambda)^{1 / \alpha}\left(\ln \frac{2 N}{n}+\frac{1}{\alpha}\right)^{1 / \alpha} \leq\left(\frac{C^{\prime}}{\alpha}\right)^{1 / \alpha}(n N)^{1 / 4}
$$

and

$$
\beta \leq \frac{1}{(10 N)^{4}} \exp \left(\frac{4 n^{\alpha / 2}}{(3.5 \ln (2 n))^{2 \alpha}}\right)+\frac{N^{2}}{2 \exp \left((2 n N)^{\alpha / 4}\right)} \leq 1 / 64,
$$

provided that $N \geq(4 / \alpha)^{8 / \alpha}$. Thus with probability at least $1-\sqrt{\beta}$ we have

$$
A_{n}^{2} \leq C^{\prime \prime} \max _{i \leq N}\left|X_{i}\right|^{2}+\left(\frac{C^{\prime \prime \prime}}{\alpha}\right)^{2 / \alpha} \sqrt{n N}
$$

where $C^{\prime \prime}$ and $C^{\prime \prime \prime}$ are absolute positive constants. This together with the estimate for $Z$ completes the proof (note that $C(\alpha) \leq C(2 / \alpha)^{5 / \alpha}$ ). 


\section{The proof of Theorem 2.1}

In this section we prove the main technical result of this paper, Theorem 2.1 , which establishes upper bounds for norms of submatrices of random matrices with independent columns. Recall that for $1 \leq k \leq N$ the parameters $A_{k}$ and $B_{k}$ are defined by (6) .

\subsection{Bilinear forms of independent vectors}

Let $X_{1}, \ldots X_{N}$ be independent random vectors and $a \in \mathbb{R}^{N}$. Given disjoint sets $T, S \subset\{1, \ldots, N\}$ we let

$$
Q(a, T, S)=\left|\left\langle\sum_{i \in T} a_{i} X_{i}, \sum_{j \in S} a_{j} X_{j}\right\rangle\right|
$$

with the convention that $\sum_{i \in \emptyset} a_{i} X_{i}=0$.

The following two lemmas are in the spirit of Lemma 2.3 in [25]. Recall that $\left(s_{i}^{*}\right)_{i}$ denotes a non-increasing rearrangement of $\left(\left|s_{i}\right|\right)_{i}$.

Lemma 5.1 Let $X_{1}, \ldots X_{N}$ be independent random vectors in $\mathbb{R}^{n}$. Let $\gamma \in$ $(1 / 2,1), I \subset\{1, \ldots, N\}$, and $a \in \mathbb{R}^{N}$. Let $k \geq|\operatorname{supp}(a)|$. Then there exists $\bar{a} \in \mathbb{R}^{N}$ such that $\operatorname{supp}(\bar{a}) \subset \operatorname{supp}(a),|\operatorname{supp}(\bar{a})| \leq \gamma k,|\bar{a}| \leq|a|$, and

$$
Q\left(a, I, I^{c}\right) \leq Q\left(\bar{a}, I, I^{c}\right)+\max \left\{\sum_{i=m}^{m+\ell-1} V_{i}^{*}, \sum_{i=m}^{m+\ell-1} W_{i}^{*}\right\},
$$

where $\ell=\lceil(1-\gamma) k\rceil, m=\lceil(\gamma-1 / 2) k\rceil$, and

$$
\begin{gathered}
V_{i}=\left\langle a_{i} X_{i}, \sum_{j \in I^{c}} a_{j} X_{j}\right\rangle \quad \text { for } i \in I, \\
W_{j}=\left\langle\sum_{i \in I} a_{i} X_{i}, a_{j} X_{j}\right\rangle \quad \text { for } j \in I^{c} .
\end{gathered}
$$

Proof. Let $E \subset\{1, \ldots, N\}$ be such that $\operatorname{supp}(a) \subset E$ and $|E|=k$. Everything is clear when $k=0$ or 1 , because then $Q\left(a, I, I^{c}\right)=0$. Thus we may assume that $k \geq 2$. Let $F_{1}=E \cap I$ and $F_{2}=E \cap I^{c}$. First assume that 
$s:=\left|F_{1}\right| \geq k / 2$. Note that $(1-\gamma) k \leq k / 2 \leq s$, so that $\ell \leq s$. Let $J \subset F_{1}$ be a set with $|J|=\ell$ such that the set $\left\{\left|V_{j}\right|: j \in J\right\}$ consists of $\ell$ smallest values among the values $\left\{\left|V_{i}\right|: i \in F_{1}\right\}$. (That is, $J \subset F_{1}$ is such that $|J|=\ell$ and for all $j \in J$ and $i \in F_{1} \backslash J$ we have $\left|V_{i}\right| \geq\left|V_{j}\right|$.) Now we let

$$
\bar{F}_{1}=F_{1} \backslash J \quad \text { and } \quad \bar{F}_{2}=F_{2} .
$$

Define the vector $\bar{a} \in \mathbb{R}^{N}$ by the conditions

$$
\bar{a}_{\mid \bar{F}_{1}}=a_{\mid \bar{F}_{1}}, \quad \bar{a}_{\mid \bar{J}}=0, \quad \bar{a}_{\mid \bar{F}_{2}}=a_{\mid \bar{F}_{2}} .
$$

Thus $\bar{a}$ differs from $a$ only on coordinates from $J$; in particular its support has cardinality less than or equal to $|\operatorname{supp}(a)|-|J|=s-\ell \leq k-\ell=\gamma k$. Moreover,

$$
\begin{aligned}
Q\left(a, I, I^{c}\right) & =\left|\left\langle\sum_{i \in F_{1}} a_{i} X_{i}, \sum_{j \in F_{2}} a_{j} X_{j}\right\rangle\right| \\
& \leq\left|\left\langle\sum_{i \in J} a_{i} X_{i}, \sum_{j \in F_{2}} a_{j} X_{j}\right\rangle\right|+\left|\left\langle\sum_{i \in F_{1} \backslash J} a_{i} X_{i}, \sum_{j \in F_{2}} a_{j} X_{j}\right\rangle\right| \\
& =\left|\sum_{i \in J}\left\langle a_{i} X_{i}, \sum_{j \in F_{2}} a_{j} X_{j}\right\rangle\right|+Q\left(\bar{a}, I, I^{c}\right) .
\end{aligned}
$$

Then we have

$$
\begin{aligned}
Q\left(a, I, I^{c}\right) & \leq Q\left(\bar{a}, I, I^{c}\right)+\sum_{i \in J}\left|\left\langle a_{i} X_{i}, \sum_{j \in F_{2}} a_{j} X_{j}\right\rangle\right| \\
& \leq Q\left(\bar{a}, I, I^{c}\right)+\sum_{i=s-\ell+1}^{s} V_{i}^{*} \leq Q\left(\bar{a}, I, I^{c}\right)+\sum_{i=m}^{m+\ell-1} V_{i}^{*},
\end{aligned}
$$

where $m=\lceil(\gamma-1 / 2) k\rceil$ and using that $s-\ell+1 \geq k / 2-\lceil(1-\gamma) k\rceil+1>$ $(\gamma-1 / 2) k$.

If $\left|F_{1}\right|<k / 2$ then $\left|F_{2}\right| \geq k / 2$ and we proceed similarly interchanging the role of $F_{1}$ and $F_{2}$ and obtaining

$$
Q\left(a, I, I^{c}\right) \leq Q\left(\bar{a}, I, I^{c}\right)+\sum_{i=m}^{m+\ell-1} W_{i}^{*} .
$$


Lemma 5.2 Let $\tau \geq 1$ and $X_{1}, \cdots, X_{N}$ be independent random vectors in $\mathbb{R}^{n}$ satisfying hypothesis $\mathbf{H}(\phi)$ for some function $\phi \in \mathcal{M}$ with parameter $\tau$. Let $a \in \mathbb{R}^{N}$ with $|a|=1$. In the notation of Lemma 5.1, for every $t>0$ one has

$\mathbb{P}\left(\sum_{i=m}^{m+\ell-1} U_{i}^{*}>t A_{k}\right) \leq(2 \tau)^{k}\left(\phi\left(\frac{t \sqrt{m}}{\ell}\right)\right)^{-m} \leq(2 \tau)^{k}\left(\phi\left(\frac{t \sqrt{\gamma_{0} k}}{(1-\gamma) k+1}\right)\right)^{-\gamma_{0} k}$

where $\left\{U_{i}\right\}_{i}$ denotes either $\left\{V_{i}\right\}_{i}$ or $\left\{W_{i}\right\}_{i}$, and $\gamma_{0}=\gamma-1 / 2$.

Remarks. 1. Taking $\phi(t)=t^{p}$ for some $p>0$, we obtain that if

$$
\mathbb{P}\left(\left|\left\langle X_{i}, a\right\rangle\right| \geq t\right) \leq t^{-p}
$$

then

$$
\mathbb{P}\left(\sum_{i=m}^{m+\ell-1} U_{i}^{*}>t A_{k}\right) \leq(2 \tau)^{k}\left(\frac{t \sqrt{m}}{\ell}\right)^{-m p} .
$$

Note that the condition (13) is satisfied if

$$
\sup _{i \leq N} \sup _{a \in S^{n-1}} \mathbb{E}\left|\left\langle X_{i}, a\right\rangle\right|^{p} \leq \tau
$$

2. Taking $\phi=(1 / 2) \exp \left(x^{\alpha}\right)$ for some $\alpha>0$, we obtain that if

$$
\mathbb{P}\left(\left|\left\langle X_{i}, a\right\rangle\right| \geq t\right) \leq 2 \exp \left(-t^{\alpha}\right)
$$

then

$$
\mathbb{P}\left(\sum_{i=m}^{m+\ell-1} U_{i}^{*}>t A_{k}\right) \leq(2 \tau)^{k+m} \exp \left(-m\left(\frac{t \sqrt{m}}{\ell}\right)^{\alpha}\right) .
$$

Note that the condition (15) is satisfied if

$$
\sup _{i \leq N} \sup _{a \in S^{n-1}} \mathbb{E} \exp \left(\left|\left\langle X_{i}, a\right\rangle\right|^{\alpha}\right) \leq 2 \tau
$$

Proof. Without loss of generality assume that $U_{i}=V_{i}$ for every $i$. Then

$$
\sum_{i=m}^{m+\ell-1} V_{i}^{*} \leq \ell V_{m}^{*}
$$


Let $F_{1}=\operatorname{supp}(a) \cap I$ and $F_{2}=\operatorname{supp}(a) \cap I^{c}$. Note that $V_{m}^{*}>s$ means that there exists a set $F \subset F_{1}$ of cardinality $m$ such that $V_{i}>s$ for every $i \in F$ (if cardinality of $F_{1}$ is smaller than $m$, the estimate for probability is trivial). Since $\left|F_{1}\right| \leq k$, we obtain

$\mathbb{P}\left(\sum_{i=m}^{m+\ell-1} V_{i}^{*}>t A_{k}\right) \leq \mathbb{P}\left(\ell V_{m}^{*}>t A_{k}\right) \leq\left(\begin{array}{c}k \\ m\end{array}\right) \max _{\substack{F \subset F_{1} \\|F|=m}} \mathbb{P}\left(\forall i \in F:\left|V_{i}\right|>\frac{t A_{k}}{\ell}\right)$

Denote $Z:=\sum_{j \in F_{2}} a_{j} X_{j}$. Since $|a| \leq 1$ then $|Z| \leq A_{k}$, and note that the $X_{i}$ 's, $i \in F_{1}$ are independent of $Z$. Thus, conditioning on $Z$ we obtain

$$
\begin{aligned}
\mathbb{P}\left(\sum_{i=m}^{m+\ell-1} V_{i}^{*}>t A_{k}\right) & \leq 2^{k} \max _{\substack{F \subset F_{1} \\
|F|=m}} \prod_{i \in F} \mathbb{P}\left(\left|a_{i}\right|\left|\left\langle X_{i}, Z\right\rangle\right|>\frac{t A_{k}}{\ell}\right) \\
& \leq(2 \tau)^{k} \max _{\substack{F \subset F_{1} \\
|F|=m}} \prod_{i \in F}\left(\phi\left(\frac{t}{\ell\left|a_{i}\right|}\right)\right)^{-1} .
\end{aligned}
$$

Now we show that for every $s>0$,

$$
\prod_{i \in F}\left(\phi\left(\frac{s}{\left|a_{i}\right|}\right)\right)^{-1} \leq(\phi(s \sqrt{m}))^{-m}
$$

Indeed, this estimate is equivalent to

$$
\frac{1}{m} \sum_{i \in F} \ln \phi\left(\frac{s}{\left|a_{i}\right|}\right) \geq \ln \phi(s \sqrt{m})
$$

which holds by convexity of $\ln \phi(1 / \sqrt{x})$, the facts that $|a| \leq 1$ and $|F|=m$, and since $\phi$ is increasing. Taking $s=t / \ell$, we obtain

$$
\mathbb{P}\left(\sum_{i=m}^{m+\ell-1} V_{i}^{*}>t A_{k}\right) \leq(2 \tau)^{k}(\phi(t \sqrt{m} / \ell))^{-m}
$$

Finally note that $m=\lceil(\gamma-1 / 2) k\rceil \geq \gamma_{0} k$ and $\ell=\lceil(1-\gamma) k\rceil \leq(1-\gamma) k+1$. Since $\phi$ is increasing, we obtain the last inequality, completing the proof. 


\subsection{Estimates for off-diagonal of bilinear forms}

For $1 \leq k \leq N$ and $I \subset\{1, \ldots, N\}$ we define $Q_{k}(I)$ by

$$
Q_{k}(I)=\sup _{\substack{E \subset\{1, \ldots, N\} \\|E| \leq k}} \sup _{a \in B_{2}^{E}} Q\left(a, E \cap I, E \cap I^{c}\right) .
$$

Lemmas 5.1, 5.2 and 4.1 imply the following proposition.

Proposition 5.3 Let $\tau \geq 1$ and $X_{1}, \cdots, X_{N}$ be independent random vectors in $\mathbb{R}^{n}$ satisfying hypothesis $\mathbf{H}(\phi)$ with parameter $\tau$ for some function $\phi \in \mathcal{M}$. Let $\varepsilon \in(0,1 / 2), 2 \leq k \leq N, I \subset\{1, \ldots, N\}, \gamma \in(1 / 2,1)$, and $\gamma_{0}=\gamma-1 / 2$. Then for every $t>0$ one has

$\mathbb{P}\left(Q_{k}(I)>\frac{Q_{[\gamma k]}(I)+t A_{k}}{1-2 \varepsilon}\right) \leq \exp \left(k\left(\ln \frac{5 \tau e N}{k \varepsilon}-\gamma_{0} \ln \phi\left(\frac{t \sqrt{\gamma_{0} k}}{(1-\gamma) k+1}\right)\right)\right)$.

Moreover, letting $M=\max _{i}\left|X_{i}\right|$ one has, for all $\ell>1$ and $t>0$,

$$
\mathbb{P}\left(Q_{\ell}(I)>t M\right) \leq \frac{N^{2} \tau}{4 \phi(4 t / \ell)} .
$$

Proof. For every $E \subset 1, \ldots, N$ with $|E|=k$ let $\mathcal{N}_{E}$ be an $\varepsilon$-net in $B_{2}^{E}$ of cardinality at most $(2.5 / \varepsilon)^{k}$. Let $\mathcal{N}$ denote the union of $\mathcal{N}_{E}$ 's. Lemma 4.1 yields

$$
Q_{k}(I) \leq(1-2 \varepsilon)^{-1} \sup _{\substack{E \subset\{1, \ldots, N\} \\|E| \leq k}} \sup _{a \in \mathcal{N}_{E}} Q\left(a, E \cap I, E \cap I^{c}\right)
$$

Therefore, applying Lemmas 5.1 and 5.2 , we observe that the event

$$
Q_{k}(I) \leq(1-2 \varepsilon)^{-1}\left(\sup _{\substack{E \subset\{1, \ldots, N\} \\|E| \leq \gamma k}} \sup _{a \in \mathcal{N}} Q\left(a, E \cap I, E \cap I^{c}\right)+t A_{k}\right)
$$

occurs with probability at least

$$
1-\left(\begin{array}{l}
N \\
k
\end{array}\right)\left(\frac{2.5}{\varepsilon}\right)^{k}(2 \tau)^{k}\left(\phi\left(\frac{t \sqrt{\gamma_{0} k}}{(1-\gamma) k+1}\right)\right)^{-\gamma_{0} k}
$$

This implies the first estimate. 
Now we prove the "moreover" part. For every $E \subset\{1, \ldots, N\}$ of cardinality $\ell$ denote $F_{1}=E \cap I, F_{2}=E \cap I^{c}, m=\left|F_{1}\right|$ (so $\left|F_{2}\right|=\ell-m$ ). We also denote

$$
M_{0}:=\max _{i \in I} \max _{j \in I^{c}}\left|\left\langle X_{i}, X_{j}\right\rangle\right| \text { and } \quad M_{1}:=\max _{j \in I^{c}}\left|X_{j}\right|
$$

Then for any $a \in B_{2}^{E}$ we have

$$
\begin{gathered}
\left|\left\langle\sum_{i \in F_{1}} a_{i} X_{i}, \sum_{j \in F_{2}} a_{j} X_{j}\right\rangle\right| \leq\left|\sum_{i \in F_{1}} a_{i} \sum_{j \in F_{2}} a_{j}\right| M_{0} \\
\leq \sqrt{m(\ell-m)}\left(\sum_{i \in F_{1}} a_{i}^{2}\right)^{1 / 2}\left(\sum_{j \in F_{2}} a_{j}^{2}\right)^{1 / 2} M_{0} \leq \frac{\ell}{2} \frac{M_{0}}{2} .
\end{gathered}
$$

Therefore, by the union bound,

$$
\begin{aligned}
\mathbb{P}\left(Q_{\ell}(I)>t M_{1}\right) & \leq \mathbb{P}\left(M_{0}>4 t M_{1} / \ell\right) \\
& \leq \sum_{i \in I} \sum_{j \in I^{c}} \mathbb{P}\left(\left|\left\langle X_{i}, X_{j}\right\rangle\right|>4 t M_{1} / \ell\right) .
\end{aligned}
$$

Finally, using the fact that $X_{i}$ is independent of $X_{j}$ for $i \neq j,\left|X_{j}\right| \leq M_{1}$ for every $j \in I^{c}$, and using the tail behavior of variables $\left\langle X_{i}, z\right\rangle$, we obtain

$$
\mathbb{P}\left(Q_{\ell}(I)>t M\right) \leq \mathbb{P}\left(Q_{\ell}(I)>t M_{1}\right) \leq \frac{|I|\left|I^{c}\right|}{\phi(4 t / \ell)} \leq \frac{N^{2} \tau}{4 \phi(4 t / \ell)} .
$$

Proposition 5.4 Let $1 \leq k \leq N$. Let $\tau \geq 1$ and $X_{1}, \cdots, X_{N}$ be independent random vectors in $\mathbb{R}^{n}$ satisfying hypothesis $\mathbf{H}(\phi)$ with parameter $\tau$ for some function $\phi \in \mathcal{M}$. Let $t>0, \lambda \geq 1$.

Case 1. Let $p>4$ and $\phi(x)=x^{p}$. Let $\sigma \in(2, p / 2)$. Then

$$
Q_{k}(I) \leq e^{4}\left(t \max _{i \leq N}\left|X_{i}\right|+C_{2}(\sigma, \lambda, p) \sqrt{k}\left(\frac{5 \tau e N}{k}\right)^{\sigma / p} A_{k}\right)
$$


occurs with probability at least

$$
1-\left(\frac{2(\sigma+\lambda)}{5 \tau e N(\sigma-2)}\right)^{\lambda} \frac{1}{2 \lambda-1}-\frac{N^{2} \tau(\sigma+\lambda)^{p}}{4(2 t(\sigma-2))^{p}}
$$

and

$$
C_{2}(\sigma, \lambda, p)=8 \sqrt{\frac{\sigma+\lambda}{1+\lambda / 2}}\left(\frac{2 p}{p-2 \sigma}\right)^{1+2 \sigma / p}\left(\frac{2(\sigma+\lambda)}{\sigma-2}\right)^{2 \sigma / p}
$$

Case 2. Assume that $\phi(x)=(1 / 2) \exp \left(x^{\alpha}\right)$ for some $\alpha>0$. Then for every $t>0$,

$Q_{k}(I) \leq C^{1 / \alpha}\left(t \max _{i \leq N}\left|X_{i}\right|+(C \lambda)^{1 / \alpha} \sqrt{k}\left(\left(\ln \frac{20 \tau e N}{k}\right)^{1 / \alpha}+\left(\frac{1}{\alpha}\right)^{1 / \alpha}\right) A_{k}\right)$

with probability at least

$$
1-\frac{1}{(10 \tau N)^{\lambda}} \exp \left(-\frac{\lambda k^{\alpha / 2}}{(3.5 \ln (2 k))^{2 \alpha}}\right)-\frac{N^{2} \tau}{2 \exp \left((2 t)^{\alpha}\right)} .
$$

Proof. Let $\gamma \in(1 / 2,1)$ to be chosen later. For integers $s \geq 0$ denote $k_{0}=k$, $k_{s+1}=\left[\gamma k_{s}\right]$. Clearly, the sequence is strictly decreasing whenever $k_{s} \geq 1$ and $k_{s} \leq \gamma^{s} k$. Assume that $k \geq 1 /(1-\gamma)$. Define $m$ to be the largest integer $m \geq 1$ such that $k_{m-1} \geq 1 /(1-\gamma)$. Note that $\gamma k_{m-1} \geq 1$. Therefore

$$
1 \leq k_{m}<\frac{1}{1-\gamma} \leq k_{m-1}
$$

By Proposition 5.3 we observe that for every positive $t_{s}$ and $\varepsilon_{s} \in(0,1 / 2)$, $0 \leq s \leq m$, the event

$$
Q_{k}(I) \leq\left(Q_{k_{m}}(I)+\sum_{s=0}^{m-1} t_{s} A_{k_{s}}\right) \prod_{s=0}^{m-1}\left(1-2 \varepsilon_{s}\right)^{-1}
$$

occurs with probability at least

$$
1-2 \sum_{s=0}^{m-1} \exp \left(k_{s}\left(\ln \frac{5 \tau e N}{k_{s} \varepsilon_{s}}-\gamma_{0} \ln \phi\left(\frac{t_{s} \sqrt{\gamma_{0} k}}{(1-\gamma) k+1}\right)\right)\right)
$$


Let $\varepsilon>0$ and a positive decreasing sequence $\left(\varepsilon_{s}\right)_{s}$ be chosen later and set

$$
t_{s}=\frac{(1-\gamma) k_{s}+1}{\sqrt{\gamma_{0} k_{s}}} \phi^{-1}\left(\left(\frac{5 \tau e N}{k_{s} \varepsilon_{s}}\right)^{(1+\varepsilon) / \gamma_{0}}\right)
$$

where $\phi^{-1}(s)=\min \{t \geq 0: \phi(t) \geq s\}$.

We start estimating $Q_{k}(I)$. Since $\ln (1-x) \geq-2 x$ on $(0,3 / 4]$, we observe that for $\varepsilon_{s}<3 / 8$,

$$
\sum_{s=0}^{m-1} \ln \left(1-2 \varepsilon_{s}\right) \geq \sum_{s=0}^{m-1}-4 \varepsilon_{s}
$$

so that

$$
\prod_{s=0}^{m-1}\left(1-2 \varepsilon_{s}\right)^{-1} \leq \exp \left(4 \sum_{s=0}^{m-1} \varepsilon_{s}\right)
$$

Note that

$$
\sum_{s=0}^{m-1} t_{s} A_{k_{s}} \leq A_{k} \sum_{s=0}^{m-1} t_{s}
$$

Thus by (20) and by our choice of $t_{s}$,

$$
Q_{k}(I) \leq \exp \left(4 \sum_{s=0}^{m-1} \varepsilon_{s}\right)\left(Q_{k_{m}}(I)+A_{k} \sum_{s=0}^{m-1} t_{s}\right)
$$

with probability at least

$$
1-2 \sum_{s=0}^{m-1} \exp \left(-k_{s} \varepsilon \ln \frac{5 \tau e N}{k_{s} \varepsilon_{s}}\right) \geq 1-2 \exp \left(-k_{m-1} \varepsilon \ln \frac{5 \tau e N}{k_{m-1}}\right) \sum_{s=0}^{m-1} \varepsilon_{s}^{k_{s} \varepsilon} .
$$

Since $k_{m-1} \geq 1 /(1-\gamma)$, this probability is larger than

$$
1-2 \exp \left(-\frac{\varepsilon}{1-\gamma} \ln (5 \tau e(1-\gamma) N)\right) \sum_{s=0}^{m-1} \varepsilon_{s}^{k_{s} \varepsilon} .
$$

Thus it is enough to choose appropriately $\varepsilon_{s}$ and to estimate $\sum_{s=0}^{m-1} t_{s}, Q_{k_{m}}(I)$ and $\sum_{s=0}^{m-1} \varepsilon_{s}^{k_{s} \varepsilon}$. We distinguish two cases for $\phi$.

Case 1: $\phi(x)=x^{p} . \quad$ In this case we choose $\varepsilon_{s}=(s+2)^{-2}$ so that

$$
\sum_{s=0}^{m-1} \varepsilon_{s}^{k_{s} \varepsilon}=\sum_{s=0}^{m-1}(s+2)^{-2 k_{s} \varepsilon} \leq \sum_{s=0}^{m-1}(s+2)^{-2 k_{m-1} \varepsilon} \leq \frac{1}{2 k_{m-1} \varepsilon-1} .
$$


Choose $\varepsilon=\lambda(1-\gamma)$. Since $\lambda \geq 1$ and $k_{m-1} \geq 1 /(1-\gamma)$, we have $2 k_{m-1} \varepsilon \geq$ $2 \varepsilon /(1-\gamma)=2 \lambda$ and

$$
\sum_{s=0}^{m-1}(s+2)^{-2 k_{s} \varepsilon} \leq \frac{1}{2 \lambda-1}
$$

Using again $k_{m-1} \geq(1-\gamma)^{-1}$, we conclude that the probability in (22) is larger than

$$
1-(5 \tau e N(1-\gamma))^{-\lambda} \frac{2}{2 \lambda-1}
$$

Now we estimate $\sum_{s=0}^{m-1} t_{s}$. We have

$t_{s}=\frac{(1-\gamma) k_{s}+1}{\sqrt{\gamma_{0} k_{s}}} \phi^{-1}\left(\left(\frac{5 \tau e N}{k_{s} \varepsilon_{s}}\right)^{(1+\varepsilon) / \gamma_{0}}\right)=\frac{(1-\gamma) k_{s}+1}{\sqrt{\gamma_{0} k_{s}}}\left(\frac{5 \tau e N}{k_{s} \varepsilon_{s}}\right)^{(1+\varepsilon) / \gamma_{0} p}$.

Recall that $\gamma>1 / 2, k_{m-1} \geq 1 /(1-\gamma)$, so that $(1-\gamma) k_{s}+1 \leq 2(1-\gamma) k_{s}$ for $s \leq m-1$. Thus

$$
t_{s} \leq \frac{2(1-\gamma) \sqrt{k}_{s}}{\sqrt{\gamma_{0}}}\left(\frac{5 \tau e N}{k_{s} \varepsilon_{s}}\right)^{(1+\varepsilon) / \gamma_{0} p}
$$

Let $b=(1+\varepsilon) / \gamma_{0} p$. Assume that $b<1 / 2$. Since $k_{s} \leq \gamma^{s} k$, we have

$$
\sum_{s=0}^{m-1} t_{s} \leq \frac{2(1-\gamma) k^{1 / 2-b}(5 \tau e N)^{b}}{\sqrt{\gamma_{0}}} \sum_{s=0}^{m-1}(s+2)^{\delta b} \gamma^{s(1 / 2-b)}
$$

Since the function $h(z)=z^{2 b} \gamma^{z(1 / 2-b)}$ on $\mathbb{R}^{+}$is first increasing and then decreasing, we get

$$
\begin{gathered}
\sum_{s=0}^{m-1}(s+2)^{2 b} \gamma^{s(1 / 2-b)}=\gamma^{-2(1 / 2-b)} \sum_{s=2}^{m+1} h(s) \leq \gamma^{-1}\left(\sup _{z>0} h(z)+\int_{0}^{\infty} h(z) d z\right) \\
\leq 2\left(\left(\frac{2 b}{(1 / 2-b) e \ln (1 / \gamma)}\right)^{2 b}+\frac{\Gamma(1+2 b)}{((1 / 2-b) \ln (1 / \gamma))^{1+2 b}}\right) .
\end{gathered}
$$

As $2 b \leq 1, \Gamma(1+2 b) \leq 1$. Using also that $\ln (1 / \gamma) \geq 1-\gamma$, we observe that the previous quantity does not exceed

$$
\frac{4}{((1 / 2-b)(1-\gamma))^{1+2 b}}
$$


Coming back to (24), we get

$$
\sum_{s=0}^{m-1} t_{s} \leq \frac{8 k^{1 / 2-b}(5 \tau e N)^{b}}{(1 / 2-b)^{1+2 b}(1-\gamma)^{2 b} \sqrt{\gamma-1 / 2}}
$$

To conclude this computation, we choose the parameter

$$
\gamma=\frac{1+\lambda+\sigma / 2}{\sigma+\lambda}
$$

Note that $\gamma \in(1 / 2,1)$ as required, since $\lambda \geq 1$ and $2<\sigma$. With such a choice of $\gamma$, we have $b=\sigma / p<1 / 2$, since $\sigma<p / 2$. Thus from (25) and (23)

$$
\sum_{s=0}^{m-1} t_{s} \leq 8 \sqrt{k}\left(\frac{5 \tau e N}{k}\right)^{\sigma / p}\left(\frac{p}{p / 2-\sigma}\right)^{1+2 \sigma / p}\left(\frac{\sigma+\lambda}{\sigma / 2-1}\right)^{2 \sigma / p} \sqrt{\frac{\sigma+\lambda}{1+\lambda / 2}}
$$

holds with probability larger than

$$
1-\left(5 \tau e N \frac{\sigma / 2-1}{\sigma+\lambda}\right)^{-\lambda} \frac{2}{2 \lambda-1}
$$

Finally, to estimate $Q_{k_{m}}$, we note that

$$
k_{m}<\frac{1}{1-\gamma}=\frac{\sigma+\lambda}{\sigma / 2-1}
$$

and apply "moreover" part of Proposition 5.3 (with $\ell=k_{m}$ ). Note that at the beginning of the proof we assumed that $k \geq 1 /(1-\gamma)$. In the case $k<$ $1 /(1-\gamma)$ the result trivially holds by the "moreover" part of Proposition 5.3 applied with $\ell=k$.

Case 2: $\phi(x)=(1 / 2) \exp \left(x^{\alpha}\right)$. In this case we choose $\gamma=2 / 3$, so that $\gamma_{0}=1 / 6$. As before we assume that $k \geq 1 /(1-\gamma)=3$ (otherwise $\left.Q_{k}(I) \leq Q_{2}(I)\right)$. By (19) we have $k_{m}<3$, hence, by (21)

$$
Q_{k}(I) \leq \exp \left(4 \sum_{s=0}^{m-1} \varepsilon_{s}\right)\left(Q_{2}(I)+A_{k} \sum_{s=0}^{m-1} t_{s}\right)
$$

We define $k_{s}$ by

$$
\varepsilon_{s}=\frac{1}{2} \exp \left(-\left(\frac{k}{k_{s}}\right)^{\alpha / 2} \frac{1}{(s+2)^{2 \alpha}}\right) .
$$


Observe that since $k_{s} \leq \gamma^{s} k$ and $\gamma=2 / 3$, one has

$$
\varepsilon_{s} \leq \frac{1}{2} \exp \left(-\left(\frac{3}{2}\right)^{\alpha s / 2} \frac{1}{(s+2)^{2 \alpha}}\right) \leq \frac{1}{2 e}(s+2)^{2 \alpha}\left(\frac{2}{3}\right)^{s \alpha / 2}
$$

which implies

$$
\sum_{s=0}^{m-1} \varepsilon_{s} \leq \frac{C}{\alpha}
$$

for a positive absolute constant $C$.

We have

$t_{s}=\sqrt{6} \frac{k_{s} / 3+1}{\sqrt{k_{s}}} \phi^{-1}\left(\left(\frac{5 \tau e N}{k_{s} \varepsilon_{s}}\right)^{6(1+\varepsilon)}\right)=\sqrt{6} \frac{k_{s} / 3+1}{\sqrt{k_{s}}}\left(\ln \left(2\left(\frac{5 \tau e N}{k_{s} \varepsilon_{s}}\right)^{6(1+\varepsilon)}\right)\right)^{1 / \alpha}$.

By (19) we have $k_{m}<3 \leq k_{m-1}$, hence,

$$
\begin{aligned}
t_{s} & \leq \sqrt{6} \frac{2}{3} \sqrt{k_{s}}(6(1+\varepsilon))^{1 / \alpha}\left(\ln \frac{20 \tau e N}{k_{s} \varepsilon_{s}}+\ln \frac{1}{2 \varepsilon_{s}}\right)^{1 / \alpha} \\
& \leq \sqrt{6} \frac{2}{3} 2^{1 / \alpha} \sqrt{k_{s}}(6(1+\varepsilon))^{1 / \alpha}\left(\left(\ln \frac{20 \tau e N}{k_{s} \varepsilon_{s}}\right)^{1 / \alpha}+\left(\ln \frac{1}{2 \varepsilon_{s}}\right)^{1 / \alpha}\right) .
\end{aligned}
$$

By the choice of $\varepsilon_{s}$ we obtain

$$
\sum_{s=0}^{m-1} \sqrt{k_{s}}\left(\ln \frac{1}{2 \varepsilon_{s}}\right)^{1 / \alpha} \leq \sqrt{k} \sum_{s=0}^{m-1}(s+2)^{-2} \leq 3 \sqrt{k}
$$

Since $3^{-s} k \leq k_{s} \leq(2 / 3)^{s} k$, we observe

$$
\begin{gathered}
\sum_{s=0}^{m-1} \sqrt{k_{s}}\left(\ln \frac{20 \tau e N}{k_{s} \varepsilon_{s}}\right)^{1 / \alpha} \leq \sqrt{k} \sum_{s=0}^{m-1}\left(\frac{2}{3}\right)^{s / 2}\left(\ln \frac{20 \tau e N 3^{s}}{k}\right)^{1 / \alpha} \\
\leq \sqrt{k}\left(\sum_{s=0}^{m-1}\left(\frac{2}{3}\right)^{s / 2} 2^{1 / \alpha}\left(\ln \frac{20 \tau e N}{k}\right)^{1 / \alpha}+\sum_{s=0}^{m-1}\left(\frac{2}{3}\right)^{s / 2}(2 s \ln 3)^{1 / \alpha}\right) \\
\leq C_{1}^{1 / \alpha} \sqrt{k}\left(\left(\ln \frac{20 \tau e N}{k}\right)^{1 / \alpha}+\Gamma(1+1 / \alpha)\right)
\end{gathered}
$$


where $C_{1}$ is an absolute positive constant and $\Gamma$ is the Gamma function. This together with (27) implies that

$$
\sum_{s=0}^{m-1} t_{s} \leq\left(C_{2}(1+\varepsilon)\right)^{1 / \alpha} \sqrt{k}\left(\left(\ln \frac{20 \tau e N}{k}\right)^{1 / \alpha}+\Gamma(1+1 / \alpha)\right),
$$

where $C_{2}$ is an absolute positive constant.

Now we estimate the probability. By the choice of $k_{s}$ we have

$$
\begin{aligned}
\sum_{s=0}^{m-1} \varepsilon_{s}^{\varepsilon k_{s}} & =\sum_{s=0}^{m-1} \exp \left(-\varepsilon k_{s} \ln \left(1 / \varepsilon_{s}\right)\right)=\sum_{s=0}^{m-1} \exp \left(-\varepsilon k_{s}\left(\ln 2+\left(k / k_{s}\right)^{\alpha / 2}(s+2)^{-2 \alpha}\right)\right) \\
& \leq \sum_{s=0}^{m-1} \exp \left(-\varepsilon k_{s}^{1-\alpha / 2} k^{\alpha / 2}(s+2)^{-2 \alpha}\right) .
\end{aligned}
$$

Since $k_{s} \geq k_{m-1} \geq 1 /(1-\gamma)$ and $s+2 \leq m+1$ for every $s \leq m-1$, we get that

$$
\sum_{s=0}^{m-1} \varepsilon_{s}^{\varepsilon k_{s}} \leq m \exp \left(-\frac{\varepsilon}{(1-\gamma)^{1-\alpha / 2}} \frac{k^{\alpha / 2}}{(m+1)^{2 \alpha}}\right) .
$$

Since $m$ is chosen such that $1 /(1-\gamma) \leq k_{m-1} \leq(2 / 3)^{m-1} k$, we observe that

$$
m-1 \leq \frac{\ln (k(1-\gamma))}{\ln (3 / 2)}
$$

Therefore,

$$
\begin{aligned}
\sum_{s=0}^{m-1} \varepsilon_{s}^{\varepsilon k_{s}} & \leq\left(1+\frac{\ln (k / 3)}{\ln (3 / 2)}\right) \exp \left(-\frac{\varepsilon}{(1 / 3)^{1-\alpha / 2}} \frac{k^{\alpha / 2}}{(2.5 \ln k)^{2 \alpha}}\right) \\
& \leq 2 \exp \left(-3 \varepsilon \frac{k^{\alpha / 2}}{3^{\alpha / 2}(2.5 \ln k)^{2 \alpha}}\right),
\end{aligned}
$$

which shows that probability in (22) is at least

$$
1-\frac{4}{(15 \tau e N)^{3 \varepsilon}} \exp \left(-3 \varepsilon \frac{k^{\alpha / 2}}{(3.5 \ln k)^{2 \alpha}}\right) .
$$

Finally, to estimate $Q_{2}(I)$ we apply the "moreover" part of Proposition 5.3 (with $\ell=2$ ). Choosing $\varepsilon=\lambda / 3$ and combining estimates (26) , and (28) with the estimate for $Q_{2}(I)$ we obtain the desired result. 


\subsection{Estimating $A_{k}$ and $B_{k}$}

We are now ready to pass to the proof of Theorem 2.1. To prove the theorem we need two simple lemmas.

Lemma 5.5 Let $\beta \in(0,1)$. Let $\mathbb{P}_{1}$ and $\mathbb{P}_{2}$ be probability measures on $\Omega_{1}$ and $\Omega_{2}$ respectively and let $V \subset \Omega_{1} \otimes \Omega_{2}$ be such that

$$
\mathbb{P}_{1} \otimes \mathbb{P}_{2}(V) \geq 1-\beta
$$

Then there exists $W \subset \Omega_{2}$ such that

$$
\mathbb{P}_{2}(W) \geq 1-\sqrt{\beta} \quad \text { and } \quad \forall x_{2} \in W, \mathbb{P}_{1}\left(\left\{x_{1}:\left(x_{1}, x_{2}\right) \in V\right\}\right) \geq 1-\sqrt{\beta}
$$

Proof. Fix some $\delta \in(0,1)$. Let

$$
W:=\left\{x_{2} \in \Omega_{2}: \mathbb{P}_{1}\left(\left\{x_{1} \in \Omega_{1}:\left(x_{1}, x_{2}\right) \in V\right\}\right) \geq 1-\delta\right\}
$$

Clearly,

$$
W^{c}=\left\{x_{2} \in \Omega_{2}: \mathbb{P}_{1}\left(\left\{x_{1} \in \Omega_{1}:\left(x_{1}, x_{2}\right) \in V^{c}\right\}\right) \geq \delta\right\}
$$

Then

$$
\begin{aligned}
\beta & \geq \mathbb{P}_{1} \otimes \mathbb{P}_{2}\left(V^{c}\right)=\int_{\Omega_{2}} \mathbb{P}_{1}\left(\left\{x_{1} \in \Omega_{1}:\left(x_{1}, x_{2}\right) \in V^{c}\right\}\right) d \mathbb{P}_{2}\left(x_{2}\right) \\
& \geq \int_{W^{c}} \mathbb{P}_{1}\left(\left\{x_{1} \in \Omega_{1}:\left(x_{1}, x_{2}\right) \in V^{c}\right\}\right) d \mathbb{P}_{2}\left(x_{2}\right) \geq \delta \mathbb{P}_{2}\left(W^{c}\right),
\end{aligned}
$$

which means $\mathbb{P}_{2}(W) \geq 1-\beta / \delta$. The choice $\delta=\sqrt{\beta}$ completes the proof.

The following lemma is obvious.

Lemma 5.6 Let $x_{1}, \ldots, x_{N} \in \mathbb{R}^{n}$, then

$$
\sum_{i \neq j}\left\langle x_{i}, x_{j}\right\rangle=2^{2-N} \sum_{I \subset\{1, \ldots, N\}} \sum_{i \in I} \sum_{j \in I^{c}}\left\langle x_{i}, x_{j}\right\rangle .
$$


Proof of Theorem 2.1. From Lemma 5.6 we have

$$
\left.|| \sum_{i=1}^{N} a_{i} X_{i}\right|^{2}-\sum_{i=1}^{N} a_{i}^{2}\left|X_{i}\right|^{2}\left|=2^{2-N}\right| \sum_{I \subset\{1,2, \ldots, N\}}\left\langle\sum_{i \in I} a_{i} X_{i}, \sum_{j \in I^{c}} a_{j} X_{j}\right\rangle .
$$

We deduce that

$$
\begin{gathered}
B_{k}^{2} \leq 2^{2-N} \sup _{a \in U_{k}} \sum_{I \subset\{1,2, \ldots, N\}} Q\left(a, I, I^{c}\right) \leq 2^{2-N} \sum_{I \subset\{1,2, \ldots, N\}} \sup _{a \in U_{k}} Q\left(a, I, I^{c}\right) \\
\leq 2^{2-N} \sum_{I \subset\{1,2, \ldots, N\}} Q_{k}(I) .
\end{gathered}
$$

Let $I \subset\{1, \ldots, N\}$ be fixed. Proposition 5.4 implies

$$
\mathbb{P}\left(Q_{k}(I) \leq M_{0}\right) \geq 1-\beta,
$$

where

$$
M_{0}:=C_{\phi} t \max _{i \leq N}\left|X_{i}\right|+\left(M_{1} / 4\right) A_{k} .
$$

Consider two probability spaces $\{I: I \subset\{1, \ldots, N\}\}$ with the normalized counting measure $\mu$ and our initial probability space $(\Omega, \mathbb{P})$, on which $X_{i}$ 's are defined. By (29) we observe that the $\mu \otimes \mathbb{P}$ probability of the event $V:=\left\{Q_{k}(I) \leq M_{0}\right\}$ is at least $1-\beta$. Then Lemma 5.5 implies that there exists $W \subset \Omega$ such that $\mathbb{P}(W) \geq 1-\sqrt{\beta}$ and such that for every $\omega \in W$ one has $\mu\left(\left\{Q_{k}(I) \leq M_{0}\right\}\right) \geq 1-\sqrt{\beta}$. Since $Q_{k}(I) \leq A_{k}^{2}$, we obtain that for every $\omega \in W$,

$$
B_{k}^{2} \leq 4 M_{0}+4 \sqrt{\beta} A_{k}^{2} .
$$

Since $A_{k}^{2} \leq \max _{i \leq N}\left|X_{i}\right|^{2}+B_{k}^{2}$, we have

$$
A_{k}^{2} \leq \frac{4 M_{0}+\max _{i \leq N}\left|X_{i}\right|^{2}}{1-4 \sqrt{\beta}} \quad \text { and } \quad B_{k}^{2} \leq \frac{4\left(M_{0}+\sqrt{\beta} \max _{i \leq N}\left|X_{i}\right|^{2}\right)}{1-4 \sqrt{\beta}} .
$$

Therefore

$$
A_{k}^{2} \leq(1-4 \sqrt{\beta})^{-1}\left(\max _{i \leq N}\left|X_{i}\right|^{2}+4 C_{\phi} t \max _{i \leq N}\left|X_{i}\right|+M_{1} A_{k}\right) .
$$

Using $\sqrt{u^{2}+v^{2}} \leq u+v$, and denoting $\gamma=(1-4 \sqrt{\beta})^{-1}$ (recall $M=$ $\left.\max _{i \leq N}\left|X_{i}\right|\right)$ we obtain

$$
A_{k} \leq \sqrt{\gamma} M+2 \sqrt{C_{\phi} \gamma t M}+\gamma M_{1}
$$


which proves the estimate for $A_{k}$. Plugging this into (30), we also observe

$$
\begin{aligned}
B_{k}^{2} & \leq \gamma\left(4 \sqrt{\beta} M^{2}+4 C_{\phi} t M+\gamma M_{1}^{2}+\sqrt{\gamma} M M_{1}+2 \sqrt{C_{\phi} \gamma t M} M_{1}\right) \\
& \leq \gamma\left(4 \sqrt{\beta} M^{2}+8 C_{\phi} t M+2 \gamma M_{1}^{2}+\sqrt{\gamma} M M_{1}\right) .
\end{aligned}
$$

This completes the proof.

\section{Optimality}

In this section we discuss optimality of estimates in Theorems 2.1 and 3.1 , In Propositions 6.5, 6.6 and 6.7 we will prove results justifying remarks on optimality following these theorems.

To obtain the lower estimates on $A_{m}$ we use the following observation.

Lemma 6.1 Let $A=\left(X_{i j}\right)_{i \leq n, j \leq N}$ be an $n \times N$ matrix with i.i.d. entries. Then

$$
\mathbb{P}\left(A_{m} \geq t\right) \geq \frac{1}{2} \quad \text { whenever } \quad \mathbb{P}\left(\left|X_{11}\right| \geq \frac{t}{\sqrt{m}}\right) \geq \frac{m+1}{N} .
$$

Proof. For every $i \leq N$, let $X_{j} \in \mathbb{R}^{n}$ be the $j$-th columns of $A$. For $m \leq N$ we have

$$
\begin{gathered}
A_{m}=\sup _{a \in U_{m}}\left|\sum_{j=1}^{N} a_{j} X_{j}\right| \geq \sup _{a \in U_{m}}\left|\sum_{j=1}^{N} a_{j} X_{1 j}\right| \geq \sup _{\substack{a \in U_{m} \\
a_{j} \in\{ \pm 1 / \sqrt{m}, 0\}}}\left|\sum_{j=1}^{N} a_{j} X_{1 j}\right| \\
=\frac{1}{\sqrt{m}} \sum_{j=1}^{m} X_{1 j}^{*} \geq \sqrt{m} X_{1 m}^{*} .
\end{gathered}
$$

Therefore, using independence, we have

$$
\mathbb{P}\left(A_{m} \geq t\right) \geq \mathbb{P}\left(X_{1 m}^{*} \geq \frac{t}{\sqrt{m}}\right)=\mathbb{P}(Y \geq m),
$$

where $Y$ is a real random variable with a binomial distribution of size $N$ and parameter $v=\mathbb{P}\left(\left|X_{11}\right| \geq \frac{t}{\sqrt{m}}\right)$. It is well known that the median of $Y$, $\operatorname{med}(Y)$ satisfies

$$
\lfloor N v\rfloor \leq \operatorname{med}(Y) \leq\lceil N v\rceil .
$$


Thus $\mathbb{P}\left(A_{m} \geq t\right) \geq \frac{1}{2}$ whenever $m \leq\lfloor N v\rfloor$. This implies the result.

To evaluate RIP, we will use the following simple observation.

Lemma 6.2 Let $n \leq N$ and $m \leq N$. Let $A$ be an $n \times N$ random matrix satisfying

$$
\mathbb{P}\left(A_{m} \geq t \sqrt{m}\right) \geq \frac{1}{2}
$$

Assume also that $A$ satisfies $R I P_{m}(\delta)$ for some $\delta<1$ with probability greater than $1 / 2$. Then

$$
m t^{2} \leq 2 n
$$

Proof. As $A$ satisfies $\operatorname{RIP}_{m}(\delta)$ for some $\delta<1$ with probability greater than $1 / 2$, then clearly

$$
A_{m}^{2}=\sup _{a \in U_{m}}\left|\sum a_{i} X_{i}\right|^{2} \leq 2 n
$$

with probability greater than $1 / 2$. Therefore, with positive probability one has

$$
t \sqrt{m} \leq A_{m} \leq \sqrt{2 n}
$$

which implies the result.

In order to show that a matrix with i.i.d. random variables satisfies condition $\mathbf{H}(\phi)$ with $\phi(t)=t^{p}$ we need the Rosenthal's inequality ([29], see also [17]). As usual, by $\|\cdot\|_{q}$ for a random variable $\xi$ we mean its $L_{q}$-norm and for an $a \in \mathbb{R}^{n}$ its $\ell_{q}$-norm, that is

$$
\|\xi\|_{q}=\left(\mathbb{E}|\xi|^{q}\right)^{1 / q} \quad \text { and } \quad\|a\|_{q}=\left(\sum_{i=1}^{n}\left|a_{i}\right|^{q}\right)^{1 / q} .
$$

Note that originally the Rosenthal inequality was proved for symmetric random variables, but using standard symmetrization argument (i.e., passing from random variables $\xi_{i}$ 's to $\left(\xi_{i}-\xi_{i}^{\prime}\right)^{\prime}$ 's, where $\left(\xi_{i}^{\prime}\right)^{\text {'s }}$ have the same distribution and are independent), one can pass to centered random variables.

Lemma 6.3 Let $q>2$ and $a \in \mathbb{R}^{n}$. Let $\xi_{1}, \ldots, \xi_{n}$ be i.i.d. centered random variables with finite $q$-th moment. Then there exists a positive absolute constant $C$ such that

$$
\frac{1}{2} M_{q} \leq\left\|\sum_{i=1}^{n} a_{i} \xi_{i}\right\|_{q} \leq C \frac{q}{\ln q} M_{q}
$$


where $M_{q}:=\max \left\{\|a\|_{2}\left\|\xi_{1}\right\|_{2},\|a\|_{q}\left\|\xi_{1}\right\|_{q}\right\}$.

The following is an almost immediate corollary of Rosenthal's inequality. It should be compared with Proposition 1.3 of [31].

Corollary 6.4 Let $p>4$. Let $\xi$ be a random variable of variance one and with a finite $p$-th moment. Let $\xi_{i j}, i \leq n, j \leq N$ be i.i.d. random variables distributed as $\xi$. Then for every $t>0$,

$$
\mathbb{P}\left(\max _{j \leq N}\left|\frac{1}{n} \sum_{i=1}^{n} \xi_{i j}^{2}-1\right|>t\right) \leq\left(\frac{C p}{t \ln p}\right)^{p / 2} \mathbb{E}|\xi|^{p} \frac{N}{n^{p / 4}},
$$

where $C$ is a positive absolute constant.

Proof. Let $\xi_{1}, \ldots, \xi_{n}$ be i.i.d. random variables distributed as $\xi$. We apply Rosenthal's inequality to random variables $\left(\xi_{i}^{2}-1\right)$ with $q=p / 2$ and $a=$ $(1,1, \ldots, 1)$. Then

$$
\left\|\sum_{i=1}^{n}\left(\xi_{i}^{2}-1\right)\right\|_{p / 2} \leq C_{p} \sqrt{n}\left\|\xi^{2}-1\right\|_{p / 2} \leq C_{p} \sqrt{n}\left(\left\|\xi^{2}\right\|_{p / 2}+1\right) \leq 2 C_{p} \sqrt{n}\|\xi\|_{p}^{2},
$$

where $C_{p}=C p / \ln p$ for an absolute positive constant $C$. Using Chebyshev's inequality we observe

$$
\mathbb{P}\left(\left|\frac{1}{n} \sum_{i=1}^{n} \xi_{i}^{2}-1\right|>t\right) \leq \frac{\mathbb{E} \sum_{i=1}^{n}\left|\xi_{i}^{2}-1\right|^{p / 2}}{(t n)^{p / 2}} \leq \frac{\left(2 C_{p}\right)^{p / 2}\|\xi\|_{p}^{p}}{t^{p / 2} n^{p / 4}} .
$$

The result follows by the union bound.

As is mentioned in remarks on optimality following Theorem 2.1 the next proposition gives a lower bound for $A_{m}$ to be compared with Case 1 of Theorem 2.1

Proposition 6.5 Let $p>2,1 \leq m \leq N$. There exists a sequence of independent random vectors $X_{1}, \cdots, X_{N}$ in $\mathbb{R}^{n}$ satisfying

$$
\forall 1 \leq i \leq N \forall a \in S^{n-1} \quad \mathbb{E}\left|\left\langle X_{i}, a\right\rangle\right|^{p} \leq 1
$$

and such that

$$
\mathbb{P}\left(A_{m} \geq \frac{C p}{\ln p} \sqrt{m}\left(\frac{N}{m}\right)^{1 / p}\left(\ln \left(\frac{2 N}{m}\right)\right)^{-1 / p}\right) \geq \frac{1}{2},
$$

where $C$ is an absolute positive constant. 
Proof. Let $\lambda \geq 1$ to be set later and let us put

$$
f_{p}(x)= \begin{cases}\frac{p}{2\left(1-\lambda^{-p}\right)|x|^{p+1}} & \text { if } 1 \leq|x| \leq \lambda \\ 0 & \text { otherwise }\end{cases}
$$

We have $\int f_{p}(x) d x=1$ and

$$
a_{p}^{p}:=\int|x|^{p} f_{p}(x) d x=p \frac{\ln \lambda}{1-\lambda^{-p}} .
$$

Consider the random variable $\xi(\omega)=\omega$ with respect to the density $f_{p}$ and let $\left(X_{i j}\right)$ be i.i.d. copies of $\xi / a_{p}$. Clearly, $\mathbb{E}\left|X_{11}\right|^{p}=1$. Since, for $s \in[1, \lambda]$

$$
\mathbb{P}(|\xi|>s)=\frac{1}{1-\lambda^{-p}}\left(\frac{1}{s^{p}}-\frac{1}{\lambda^{p}}\right),
$$

a short computation using (31) shows that $\mathbb{P}\left(A_{m} \geq t\right) \geq \frac{1}{2}$ provided that

$$
\begin{aligned}
t \leq & \left(\frac{1-\lambda^{-p}}{p \ln \lambda}\right)^{1 / p} \sqrt{m}\left(\frac{N}{(m+1)\left(1-\lambda^{-p}\right)+N \lambda^{-p}}\right)^{1 / p} \\
& =\sqrt{m}\left(\frac{1}{p \ln \lambda}\right)^{1 / p}\left(\frac{N}{m+1+N /\left(\lambda^{p}-1\right)}\right)^{1 / p} .
\end{aligned}
$$

Choosing $\lambda$ from $\lambda^{p}-1=N /(m+1)$, we obtain $\mathbb{P}\left(A_{m} \geq t\right) \geq \frac{1}{2}$ provided that

$$
t \leq \sqrt{m}\left(\frac{N}{2(m+1) \ln (2 N /(m+1))}\right)^{1 / p} .
$$

Finally, to satisfy condition (33), we pass from matrix $A$ to $A^{\prime}=A / c_{p}=$ $\left(X_{i j} / c_{p}\right)_{i j}$, where $c_{p} \leq C p / \ln p$ is a constant in Rosenthal's inequality (32). By Rosenthal's inequality, the sequence of columns of $A^{\prime}$ satisfies the condition (33).

The next proposition gives an upper bound on the size of sparsity $m$ in order to satisfy RIP under condition of Case 1 of Theorem 3.1 (see Remark 3 following this theorem). 
Proposition 6.6 Let $q>p>2, n \leq N$ and $m \leq N$. There exist an absolute positive constant $C$, an $n \times N$ matrix $A$, whose columns $X_{1}, \ldots, X_{N}$ are independent random vectors satisfying

$$
\forall 1 \leq i \leq N \forall a \in S^{n-1} \quad \mathbb{E}\left|\left\langle X_{i}, a\right\rangle\right|^{p} \leq\left(\frac{C p}{\ln p}\right)^{p} \frac{q}{q-p}\left(\frac{q-2}{q}\right)^{p / 2},
$$

and for every $t \in(0,1)$,

$$
\mathbb{P}\left(\max _{i \leq N}\left|\frac{\left|X_{i}\right|^{2}}{n}-1\right| \geq t\right) \leq t^{p / 2}
$$

provided that

$$
N \leq\left(\frac{q \ln p}{C(q-2) p}\right)^{p / 2} \frac{q-p}{q} t^{p} n^{p / 4} .
$$

Assume that A satisfies $R I P_{m}(\delta)$ for some $\delta<1$ with probability greater than $1 / 2$. Then

$$
m\left(\frac{N}{m+1}\right)^{2 / q} \leq \frac{2(q-2)}{q} n .
$$

Proof. Consider the density

$$
f(x)= \begin{cases}\frac{q}{2|x|^{q+1}} & \text { if }|x| \geq 1 \\ 0 & \text { otherwise. }\end{cases}
$$

We have $\int f(x) d x=1$,

$$
\int|x|^{p} f(x) d x=\frac{q}{q-p} \quad \text { and } \quad a_{2}^{2}:=\int|x|^{2} f(x) d x=\frac{q}{q-2} .
$$

Consider the random variable $\xi(\omega)=\omega$ with respect to the density $f$ and let $\left(X_{i j}\right)_{i j}$ be i.i.d. copies of $\xi / a_{2}$. Clearly,

$$
\mathbb{E}\left|X_{11}\right|^{2}=1 \quad \text { and } \quad \mathbb{E}\left|X_{11}\right|^{p}=\frac{q}{q-p}\left(\frac{q-2}{q}\right)^{p / 2} .
$$

Then Rosenthal's inequality (32) implies the condition (34) and Corollary 6.4 implies (35). 
Now we estimate $A_{m}$ for the matrix $A$, whose columns are $\left(X_{i j}\right)_{i}, j \leq N$. Since, for $s \geq 1, \mathbb{P}(|\xi|>s)=s^{-q}$, by (31), we obtain that $\mathbb{P}\left(A_{m} \geq t\right) \geq \frac{1}{2}$ provided that

$$
t \leq \sqrt{m} \sqrt{\frac{q-2}{q}}\left(\frac{N}{m+1}\right)^{1 / q} .
$$

This means

$$
\mathbb{P}\left(A_{m} \geq \sqrt{m} \sqrt{\frac{q-2}{q}}\left(\frac{N}{m+1}\right)^{1 / q}\right) \geq \frac{1}{2},
$$

and we complete the proof applying Lemma 6.2.

The next proposition shows the optimality (up to absolute constants) of the sparsity parameter in Case 2 of Theorem 3.1 (see Remark 4 following this theorem) as well as optimality of bounds for $A_{m}$ in Case 2 of Theorem 2.1 (see remarks on optimality following this theorem).

Proposition 6.7 There exist absolute positive constants $c, C$ such that the following holds. Let $\alpha \in[1,2], 1 \leq m \leq N / 2$ and $n$ satisfies $N \leq \exp \left(c n^{\alpha / 2}\right)$. There exists an $n \times N$ matrix $A$, whose columns $X_{1}, \ldots, X_{N}$ are independent random vectors satisfying

$$
\forall 1 \leq i \leq N \forall a \in S^{n-1} \quad \mathbb{E} \exp \left(\left|\left\langle X_{i}, a\right\rangle\right|^{\alpha}\right) \leq C
$$

and

$$
\mathbb{P}\left(\max _{i \leq N}\left|\frac{\left|X_{i}\right|^{2}}{n}-1\right| \geq \frac{\sqrt{2}-1}{2}\right) \leq 2 \exp \left(-c n^{\alpha / 2}\right),
$$

and such that

$$
\mathbb{P}\left(A_{m} \geq \sqrt{\frac{m}{2}}\left(\ln \frac{N}{m+1}\right)^{1 / \alpha}\right) \geq \frac{1}{2} .
$$

Additionally, if $n \leq N$ and if $A$ satisfies $R I P_{m}(\delta)$ for some $\delta<1$ with probability greater than $1 / 2$, then

$$
m\left(\ln \frac{N}{m+1}\right)^{2 / \alpha} \leq 4 n
$$

Proof. We consider a symmetric random variable $\xi$ with the distribution defined by $\mathbb{P}(|\xi|>t)=\exp \left(-t^{\alpha}\right)$. It is easy to check that

$$
\mathbb{E} \exp \left(|\xi|^{\alpha} / 2\right)=2
$$


and

$$
a:=\mathbb{E} \xi^{2}=\Gamma\left(\frac{2}{\alpha}+1\right) \in[1,2] .
$$

Let $X_{i j}, i \leq n, j \leq N$ be i.i.d. copies of $\xi / \sqrt{a}, A=\left(X_{i j}\right)_{i j}$ and $X_{j}$ 's be its columns. Applying Lemma 3.4 from [4] (see also Theorem 1.2.8 in [11]) we observe that $X_{i}$ 's satisfy conditions (36) and (37). By (31) we observe that $\mathbb{P}\left(A_{m} \geq t\right) \geq \frac{1}{2}$ provided that

$$
\mathbb{P}\left(|\xi| \geq \frac{\sqrt{a} t}{\sqrt{m}}\right)=\exp \left(-(\sqrt{a} t / \sqrt{m})^{\alpha}\right) \geq \frac{m+1}{N} .
$$

Thus it is enough to take

$$
t \leq \sqrt{\frac{m}{a}}\left(\ln \frac{N}{m+1}\right)^{1 / \alpha} .
$$

This proves the estimate (38).

Finally, the "additionally" part follows by Lemma 6.2

\section{References}

[1] R. Adamczak, O. Guédon, A.E. Litvak, A. Pajor, N. Tomczak-Jaegermann, Smallest singular value of random matrices with independent columns, C. R. Math. Acad. Sci. Paris 346 (2008), no. 15-16, 853-856.

[2] R. Adamczak, A.E. Litvak, A. Pajor, N. Tomczak-Jaegermann, Quantitative estimates of the convergence of the empirical covariance matrix in log-concave Ensembles, J. Amer. Math. Soc. 23 (2010), no. 2, 535-561.

[3] R. Adamczak, A.E. Litvak, A. Pajor, N. Tomczak-Jaegermann, Sharp bounds on the rate of convergence of empirical covariance matrix, C.R. Math. Acad. Sci. Paris, 349 (2011), 195-200.

[4] R. Adamczak, A. Litvak, A. Pajor, N. Tomczak-Jaegermann, Restricted isometry property of matrices with independent columns and neighborly polytopes by random sampling, Constructive Approximation, 34 (2011), 61-88.

[5] R. Adamczak, R. Latala, A. Litvak, A. Pajor, N. Tomczak-Jaegermann, Geometry of log-concave Ensembles of random matrices and approximate reconstruction, C.R. Math. Acad. Sci. Paris, 349 (2011), 783-786.

[6] Z.D. Bai and Y.Q. Yin, Limit of the smallest eigenvalue of a large dimensional sample covariance matrix, Ann. Probab. 21 (1993), 1275-1294. 
[7] Z.D. Bai, J. Silverstein, Y.Q. Yin, A note on the largest eigenvalue of a large dimensional sample covariance matrix, Journal of Multivariate Analysis, 26 (1988), $166-168$.

[8] J. Bourgain, Random points in isotropic convex sets, Math. Sci. Res. Inst. Publ., 34, 53-58, Cambridge Univ. Press, Cambridge (1999).

[9] E.J. Candés, J. Romberg, T. Tao, Stable signal recovery from incomplete and inaccurate measurements, Comm. Pure Appl. Math., 59 (2006), 1207-1223.

[10] E.J. Candés, T. Tao, Decoding by linear programming, IEEE Trans. Inform. Theory, 51 (2005), 4203-4215.

[11] D. Chafaï, O. Guédon, G. Lecué, A. Pajor, Interactions between compressed sensing random matrices and high dimensional geometry, Panoramas et Synthèses [Panoramas and Syntheses], 37. Soc. Math. de France, Paris, 2012.

[12] D.L. Donoho, Compressed sensing, IEEE Trans. Information Theory 52 (2006), 12891306 .

[13] A. Edelman, Eigenvalues and condition numbers of random matrices, SIAM J. of Matrix Anal. and Applic., 9 (1988), 543-560.

[14] S. van de Geer, A. Muro, On higher order isotropy conditions and lower bounds for sparse quadratic forms, Electron. J. Statist., 8 (2014), 3031-3061.

[15] O. Guédon, A.E. Litvak, A. Pajor, N. Tomczak-Jaegermann Restricted isometry property for random matrices with heavy tailed columns, C.R. Math. Acad. Sci. Paris, 352 (2014), 431-434.

[16] O. Guédon, E. Milman, Interpolating thin-shell and sharp large-deviation estimates for isotropic log-concave measures, Geom. Funct. Anal., 21 (2011), 1043-1068.

[17] W.B. Johnson, G. Schechtman, J. Zinn, Best constants in moment inequalities for linear combinations of independent and exchangeable random variables, Ann. Probab. 13 (1985), 234-253.

[18] B. Klartag, Power-law estimates for the central limit theorem for convex sets, $J$. Funct. Anal. 245 (2007), 284-310.

[19] V. Koltchinskii, S. Mendelson, Bounding the smallest singular value of a random matrix without concentration, Preprint.

[20] G. Lecué, S. Mendelson, Sparse recovery under weak moment assumptions, Journal of EMS, to appear.

[21] M. Ledoux, M. Talagrand, Probability in Banach Spaces. Isoperimetry and processes, Springer-Verlag, Berlin, 1991.

[22] A.E. Litvak, S. Spektor, Quantitative version of a Silversteins result, GAFA, Lecture Notes in Math., 2116, Springer, 2014., 335-340.

[23] S. Mendelson, A. Pajor, N. Tomczak-Jaegermann, Reconstruction and subgaussian operators, Geometric and Functional Analysis, 17 (2007), 1248-1282. 
[24] S. Mendelson, G. Paouris, On generic chaining and the smallest singular value of random matrices with heavy tails, Journal of Functional Analysis, 262 (2012), 37753811.

[25] S. Mendelson, G. Paouris, On singular values of matrices, Journal of EMS, 16 (2014), 823-834.

[26] R.I. Oliveira, The lower tail of random quadratic forms, with applications to ordinary least squares and restricted eigenvalue properties, arXiv:1312.2903.

[27] G. Paouris, Concentration of mass on convex bodies, Geom. Funct. Anal. 16, no. 5 (2006), 1021-1049.

[28] G. Paouris, Small ball probability estimates for log-concave measures, Trans. Amer. Math. Soc., 364 (2012), 287-308.

[29] H. Rosenthal, On the subspaces of $L_{p}(p>2)$ spanned by sequences of independent random variables, Israel J. Math. 8 (1970), 273-303.

[30] J. Silverstein, On the weak limit of the largest eigenvalue of a large dimensional sample covariance matrix, J. of Multivariate Anal., 30 (1989), 307-311.

[31] N. Srivastava, R. Vershynin, Covariance estimation for distributions with $2+\varepsilon$ moments, Annals of Probability 41 (2013), 3081-3111.

[32] S. Szarek, Condition numbers of random matrices, J. Complexity 7 (1991), 131-149.

[33] K.E. Tikhomirov, The smallest singular value of random rectangular matrices, with no moment assumptions on entries, Israel J. Math., to appear.

[34] R. Vershynin, How close is the sample covariance matrix to the actual covariance matrix?, J. Theoret. Probab. 25 (2012), 655-686.

[35] P. Yaskov, Lower bounds on the smallest eigenvalue of a sample covariance matrix, Electron. Commun. Probab. 19 (2014), 1-10.

Olivier Guédon, and Alain Pajor

Université Paris-Est

Laboratoire d'Analyse et Mathématiques Appliquées (UMR 8050)

UPEM, F-77454, Marne-la-Vallée, France

olivier.guedon@u-pem.fr, alain.pajor@u-pem.fr

Alexander E. Litvak and Nicole Tomczak-Jaegermann,

Dept. of Math. and Stat. Sciences,

University of Alberta,

Edmonton, Alberta, Canada, T6G 2G1.

e-mail: aelitvak@gmail.com, nicole.tomczak@ualberta.ca 